\title{
Genetic association between $H L A-G$ 14-bp polymorphism and diseases: A systematic review and meta-analysis
}

\author{
Bibiana Sgorla de Almeida ${ }^{\mathrm{a}, \mathrm{b}, 1, *}$, Yara Costa Netto Muniz ${ }^{\mathrm{c}, 1}$, Alice Heidrich Prompt ${ }^{\mathrm{c}}$, \\ Erick C. Castelli ${ }^{\mathrm{d}}$, Celso Teixeira Mendes-Junior ${ }^{\mathrm{e}}$, Eduardo Antonio Donadi ${ }^{\mathrm{a}}$ \\ a Divisão de Imunologia Clínica, Departamento de Clínica Médica, Faculdade de Medicina de Ribeirão Preto (FMRP), Universidade de São Paulo (USP), 14049-900 \\ Ribeirão Preto, SP, Brazil \\ ${ }^{\mathrm{b}}$ Laboratório Multiusuário de Estudos em Biologia, Centro de Ciências Biológicas, Universidade Federal de Santa Catarina (UFSC), Florianópolis, Brazil \\ ${ }^{\mathrm{c}}$ Departamento de Biologia Celular, Embriologia e Genética, Centro de Ciências Biológicas, Universidade Federal de Santa Catarina (UFSC), Florianópolis, Brazil \\ d Departamento de Patologia, Faculdade de Medicina de Botucatu, Unesp - Univ. Estadual Paulista, 18618-970 Botucatu, SP, Brazil \\ e Faculdade de Filosofia Ciências e Letras de Ribeirão Preto (FFCLRP), Universidade de São Paulo (USP), 14049-900 Ribeirão Preto, SP, Brazil
}

\section{A R T I C L E I N F O}

\section{Keywords:}

Non-classical MHC

HLA-G

14-bp polymorphism

Indel

3'UTR

\begin{abstract}
A B S T R A C T
Background: HLA-G is an immune checkpoint molecule. Since a differential molecule expression has been reported even for healthy individuals, many studies have focused on polymorphisms at $H L A-G$ regulatory regions, particularly the $3^{\prime}$ untranslated region (3'UTR). The presence/absence of a 14-bp sequence was the first polymorphism described and it is the most studied in association between $H L A-G$ and disorders.

Methods: In this study, we performed a systematic review and meta-analysis of all association studies published regarding the $H L A-G 14$-bp.

Results: We verified association between 14-bp alleles and diseases in the following situations: (1) presence of 14-bp (insertion) conferred susceptibility to preeclampsia (child alleles evaluated) and systemic lupus erythematosus $(\mathrm{OR}=1.42 ; 95 \% \mathrm{CI}=1.04-1.93 ; \mathrm{p}=0.026$ and $\mathrm{OR}=1.13 ; 95 \% \mathrm{CI}=1.01-1.27, \mathrm{p}=0.028) ;(2)$ 14-bp absence (deletion) was associated with increased risk to breast cancer (OR $=1.23$; $95 \% \mathrm{CI}=1.06-1.43$; $\mathrm{p}=0.006)$ and human Cytomegalovirus infection $(\mathrm{OR}=2.06 ; 95 \% \mathrm{CI}=1.60-2.64 ; \mathrm{p}<0.0001)$; and (3) a risk association was observed between the group of reproductive disorders and the 14-bp insertion $(\mathrm{OR}=1.12$; 95\%CI $=1.01-1.24 ; \mathrm{p}=0.034)$.

Conclusions: Considering that others 14-bp associations were inconclusive and that other variation sites observed at $H L A-G$ 3'UTR exhibit a proven role on post-transcriptional regulation of $H L A-G$ expression, the complete $3^{\prime}$ UTR segment should be analyzed in terms of disease susceptibility, instead of a single polymorphism.
\end{abstract}

\section{Introduction}

The non-classical human leukocyte antigen G $(H L A-G)$ gene is located at $6 \mathrm{p} 21.3$ chromosome region, within the class I major histocompatibility complex (MHC) gene cluster [1]. Among the biological properties that differentiate HLA-G from classical HLA class I (-A, -B, -C) molecules are a limited protein variability, restricted tissue expression in non-pathological conditions and modulation of cells involved on the immune response. In contrast to classical HLA molecules, HLA-G has no important role in antigen presentation, inhibits $\mathrm{CD} 8+\mathrm{T}$ and NK cell cytotoxic activity, inhibits CD4 $+\mathrm{T}$ cell proliferation and dendritic cell antigen-presentation, inhibits B cell function and induces $\mathrm{T}$ regulatory cell lines, through direct binding mainly to the ILT-2 and ILT-4 leucocyte receptors, which can also interact with classical HLA molecules, but have higher affinity for HLA-G [2,3].

Although first described in choriocarcinoma cells, the tolerogenic properties of HLA-G have been initially associated with the protection of the semi-allogeneic fetus against the maternal immune response. Today, HLA-G is considered to be an immune checkpoint molecule and its immunoregulatory functions have also been exploited in a variety of physiological and pathological conditions, for which these responses can be profitable or pernicious depending on the underlying disorder.

\footnotetext{
* Corresponding author at: Divisão de Imunologia Clínica, Departamento de Clínica Médica, Faculdade de Medicina de Ribeirão Preto (FMRP), Universidade de São Paulo (USP), 14049-900 Ribeirão Preto, SP, Brazil.

E-mail addresses: bibiana.sgorla@ufsc.br (B.S. de Almeida), yara.muniz@ufsc.br (Y.C.N. Muniz), prompt.a@ufsc.br (A.H. Prompt), castelli@fmb.unesp.br (E.C. Castelli), ctmendes@ffclrp.usp.br (C.T. Mendes-Junior), eadonadi@fmrp.usp.br (E.A. Donadi).

${ }^{1}$ These authors contributed equally for this work.
} 
For instance, in tumors, HLA-G expression can be used as an immune escape mechanism, whereas HLA-G expression by grafted tissues may delay allograft rejection [2-4].

Since the magnitude of HLA-G expression is regulated in part by the gene promoter and $3^{\prime}$ untranslated region (3'UTR), and since a differential expression of HLA-G has been reported even for healthy individuals [5], many studies have focused on the study of variable sites at the regulatory segments $[2,6]$. In contrast to classical HLA class I genes, the variability observed at the HLA-G regulatory regions is relatively higher than that observed for the coding region $[6,7]$. The nucleotide sequence of $H L A-G$ 3'UTR was first described by Geraghty (1987), encompassing approximately 1000 nucleotides and presenting several variations sites, some of them associated with distinct expression profiles $[6,7]$.

At least nine $H L A-G$ 3'UTR variation sites have polymorphic frequencies in worldwide populations, including the 14-base pair (14-bp) polymorphism (rs371194629), +3003 C/G (rs1707), +3010 G/C (rs1710), + $3027 \mathrm{C} / \mathrm{A}$ (rs17179101), + $3035 \mathrm{C} / \mathrm{T}$ (rs17179108), $+3142 \mathrm{C} / \mathrm{G}$ (rs1063320), +3187 A/G (rs9380142), +3196 C/G (rs1610696) and +3227 (rs1233331). Of these, the 14-bp, $+3142 \mathrm{C} / \mathrm{G}$ and $3187 \mathrm{~A} / \mathrm{G}$ have been functionally studied $[8,9]$, and the differential $H L A-G$ expression levels have been associated with mRNA instability, mRNA degradation by microRNAs or both mechanisms $[2,3,6]$. The presence/absence of the 14-bp fragment (14-bp insertion/deletion), located between +2961 and +2974 positions $[6,10]$, was the first polymorphic site described at the HLA-G $3^{\prime} \mathrm{UTR}$ and is the most studied one. In trophoblast samples, the presence of the 14-bp is associated with lower mRNA production for most of HLA-G membrane-bound and soluble isoforms $[11,12]$. Additionally, the presence of 14-bp is related to an alternative splicing of the $H L A-G$ primary transcript, that results in a more stable mRNA, but apparently, this higher stability does not compensate the lower HLA-G production already associated with the 14-bp insertion sequence [6]. Overall, the 14-bp insertion has been associated with lower HLA-G production and the 14-bp deletion with higher HLA-G production $[10,13]$.

Several studies have associated the 14-bp alleles with disease morbidity and disease susceptibility. Despite of the influence of the 14-bp fragment on gene expression, results are still considered inconclusive. Since all meta-analyses regarding the association between the 14-bp polymorphism have focused on particular diseases [14-22] or on a limited group of diseases [23-25], in this study we performed a systematic review of the literature followed by a meta-analysis of all association studies already published for the 14-bp polymorphism in several disorders, stratified according to groups of similar disorders, specific disorders, and even grouping disorders according to the putative beneficial or detrimental effect of the HLA-G expression.

\section{Material and Methods}

\subsection{Search strategy}

We performed a systematic review of the relevant literature using PubMed and SciELO databases up to March 2017. In our search strategy, we used four terms: "HLA-G", "Polymorphism", "3’UTR" and " $14 \mathrm{bp}$ " we also included all their entry terms registered in those databases. Between the four terms, we used the Boolean operator "AND" and between all entry terms the operator "OR". At the end, our search strategy was performed as described below: "HLA-G" OR "HLA-G Antigens" OR "HLA G Antigens" OR "HLA-G Antigen" OR "HLA G Antigen" OR "HLA G" OR "HLA-G" OR "HLA-G2 Isoform" OR "HLA G2 Isoform" OR "HLA-G2" OR "HLA-G7 Isoform" OR "HLA G7 Isoform" OR "HLA-G7" OR "HLA-G7 Antigen" OR "HLA G7 Antigen" OR "HLA-G4 Isoform" OR "HLA-G4" OR "HLA-G5 Isoform" OR "HLA-G5" OR "HLAG6 Isoform" OR "HLA G6 Isoform" OR "HLA-G6" OR "HLA-G1 Isoform" OR "HLA G1 Isoform" OR "HLA-G1" OR "HLA-G3 Isoform" OR "HLA G3 Isoform" OR "HLA-G3" AND "Polymorphism" OR "Polymorphism,
Genetic" OR "Genetic Polymorphism" OR "Polymorphism (Genetics)" OR "Genetic Polymorphisms" AND "3'UTR" OR "3' Untranslated Regions" OR " 3 'UTR” OR “ 3 'UTRs" OR " 3 ' Untranslated Region” OR " 3 ' UTR” OR “3' UTRs” AND “14 bp” OR “14-bp” OR “14 bp” OR “14 bp” OR "indel" OR "insdel".

\subsection{Inclusion and exclusion criteria}

All articles identified by database searching were screened and selected according to the inclusion and exclusion criteria. Firstly, the studies were evaluated by titles and abstracts. All studies considered irrelevant for this meta-analysis were excluded. The second stage was the article contents analysis. The full texts of the remaining articles were read to determine whether they contained information about the topic of interest. Studies were included if they met the following criteria: (1) case-control study; and (2) the presence of original data containing the 14-bp allele frequencies. We excluded: (1) studies that contained duplicated data; (2) familial studies; and (3) studies with incomplete 14-bp allele frequency data.

\subsection{Data extraction}

Data extracted from the selected articles included: (1) article title; (2) first author's name; (3) year of publication; (4) country of origin; (5) clinical situation/disease of patients; (6) genotyping method; (7) Hardy-Weinberg equilibrium (HWE) in control group; (8) number of cases and controls; (9) 14-bp insertion/deletion frequencies for cases and controls; (10) presence or not of significant association, and (11) if significant, which allele was associated. Data were extracted by two independent researchers and eventual disagreements were resolved by consensus.

\subsection{Statistical analysis}

We performed a meta-analysis for each disease/clinical situation whenever three or more published studies were available. In addition, clinical situations/diseases were grouped as follows: (1) cancer, (2) autoimmune diseases, (3) inflammatory diseases, (4) infectious diseases, (5) transplant rejection, and (6) reproductive disorders. Finally, clinical situations/diseases were organized into two distinct groups, considering the biological activity of the immune checkpoint HLA-G molecule: i) pathological conditions with hypothetical high production of HLA-G; i. e., conditions for which the a priori hypothesis was an expected association with the 14-bp deletion, reported to be associated with high HLA-G production, and ii) pathological conditions with hypothetical association with the 14-bp insertion related to low production of HLA-G.

All possible associations between the 14-bp polymorphism and clinical situations/diseases were measured by calculating a pooled Odds Ratio (OR) and 95\% confidence intervals (CI) for fixed and random effect models (FEM and REM, respectively). We used the $p$ value resulting of $\mathrm{Q}$ test, $\tau^{2}$ value and $\mathrm{I}^{2}$ value to measure between-studies heterogeneity. The effect of heterogeneity $\mathrm{I}^{2}$ ranges from 0 to $100 \%$ and represents the proportion of variability attributable to heterogeneity (values of 25,50 , and $75 \%$ were considered low, moderate, and high, respectively). The fixed-effects model assumes that a genetic factor has a similar effect on disease susceptibility across all investigated studies, and that the observed variations among the studies are caused by chance alone. While random effects model assumes that different studies show substantial diversity and assesses both within study sampling errors and study between variances. When the study groups are homogeneous, the two models are similar. In contrast, when the groups are not homogeneous, the random-effects model usually provides wider CI than the fixed-effects model. The random-effects model is best choice in the presence of significant heterogeneity between studies. Because of these, we assumed that, if $\mathrm{I}^{2}>50 \%$ or $\mathrm{Q}$ test $p$ value was $<0.01$, a 
random effect model was applied to pool data. Otherwise, a fixed and a random effect models were employed for analysis. Publication bias was evaluated by using Egger's linear regression test, which measures funnel plot asymmetry using a natural logarithm scale of ORs. If $\mathrm{p}$ value $<0.05$ indicates publication bias. Due to the defined parameters for this statistical test, we only evaluated Publication bias in those groups encompassing ten or more studies [26].

All data analyses were performed by R Statistical Software using the "meta" package. This systematic review and metanalysis was conducted according to Preferred Reporting Items for Systematic Reviews and Meta-Analysis (PRISMA) guidelines. The PRISMA checklist was included as Supplementary Material (Supplementary Material 1). The protocol for this systematic review and meta-analysis was registered at PROSPERO Systematic Review Database (PROSPERO 2017:CRD42017067176).

\subsection{HLA-G polymorphisms and proteins data collection}

We additionally analyzed the relations between the 14-bp polymorphism and others HLA-G polymorphic sites. We realized comparisons between the 14-bp polymorphism alleles and the more frequent HLA-G promoters, coding regions, 3'UTRs haplotypes and proteins. Data used for these comparisons were organized based on our own database and all available data for HLA-G 3'UTR in the 1000Genomes Project database (http://www.internationalgenome.org/).

\section{Results}

\subsection{Study identification and selection}

A total of 389 articles were identified by database searching for the systematic review. After the first screening, 172 articles were excluded after title and abstract analyses. During the second evaluation, 90 studies were excluded after further detailed assessment. All exclusions occurred on the basis of the inclusion and exclusion criteria. At the end, the remaining 125 studies were included in this meta-analysis (Fig. 1).

\subsection{Characteristics of included studies}

Articles included were published between 2001 and 2017 and all of them were written in English. Studies had been carried out in populations from 28 different countries: Australia, Brazil, Canada, China, Denmark, USA, France, Germany, Greece, India, Iran, Iraq, Ireland, Israel, Italy, Japan, Korea, Mexico, Netherlands, New Zealand, Norway, Poland, Saudi Arabia, South Africa, Spain, Taiwan, Tunisia and Zambia. The HLA-G 3'UTR 14-bp polymorphism genotyping was performed by different methodologies being the most used PCR followed by agarose or polyacrylamide gel electrophoresis (about $70 \%$ of studies). Other techniques as real time PCR and sequencing by capillary electrophoresis were also used. Articles included comprised case-control studies involving the $H L A-G$ 3'UTR 14-bp polymorphism and clinical situations/ diseases. Studies were grouped according to clinical characteristics, pathogenesis and immune response as follow: (1) cancer ${ }^{2}$ - B-Cell Lymphoma [27], Breast Cancer [28-32], Cervical Cancer [33-36], Colorectal cancer [37], Esophageal Cancer [38], Hepatocellular Carcinoma [39-41], Head and Neck Squamous-Cell Carcinoma (HNSCC) [42], Lung Cancer [43], Neuroblastoma [44], Papillary Thyroid Cancer [45] and Prostate Cancer [46]; (2) autoimmune diseases ${ }^{3}-\mathrm{Ha}$ shimoto's Thyroiditis [45], Juvenile Idiopathic Arthritis [47], Multiple Sclerosis [48-51], Non-segmental Vitiligo [52], Pemphigus Vulgaris

\footnotetext{
${ }^{2}$ Chen et al., 2012 [37] appears two times in this group, because two different populations are described in the same article.

${ }^{3}$ Veit et al., 2008 [46] appears two times in this group, because two distinct diseases data are described in the same article.
}

[53], Rheumatoid Arthritis [47,54-58], Systemic Lupus Erythematosus (LES) [59-67] and Type 1 Diabetes [68-70]; (3) inflammatory diseases $^{4}$ - Acute Coronary Syndrome [71], Atopic Asthma [72], Behcet's Disease [73,74], Celiac Disease [75,76], Coronary Disease [77,78], Crohn's Disease [79,80], Renal Disease [81,82], Type 2 Diabetes [71], and Ulcerative Colitis [79]; (4) infectious diseases - Hepatitis B Chronic Infection (HBV) [83], Helicobacter pylori Infection [84], Human Immunodeficiency Virus Infection (HIV) [85], HIV Vertical Transmission [86-89], Human T-cell Lymphotropic Virus Infection (HTLV-1) [90], Human Cytomegalovirus Infection (HCMV) [82,91,92], Human Papillomavirus Infection (HPV) [35,36,93,94], Leprosy [95] and Septic Shock [96]; (5) transplant rejection ${ }^{5}$ - Acute Allograft Rejection [82,92], Allogeneic Hematopoietic Cell Transplantation [97], Allogeneic Hematopoietic Stem Cell Transplantation (Allo-HSCT) [98], Bone Marrow Transplantation [99], Heart Transplantation [100,101], Liver transplantation [102,103] and Kidney Transplantation [104-106]; (6) reproductive disorders - Implantation Failure [107-111], Preeclampsia [112,113,122,123,114-121], Recurrent Miscarriage [107,124,133-142,125,143,126-132], Reproductive Failure $[111,144]$ and Spontaneous Miscarriage [117,134,145,146]. Additionally, two large groups were analyzed: (A) diseases/clinical situations associated with hypothetical high HLA-G expression, comprising articles about cancer, and infectious diseases; and (B) diseases/clinical situations with hypothetical low HLA-G expression, including studies about autoimmune diseases, inflammatory diseases, transplants rejection, and reproductive disorders. The characteristics of all articles included in this meta-analysis are detailed and summarized in Table 1. Additional information about the characteristics of included studies is available in Supplementary Table 1.

\subsection{Overall meta-analysis data}

For all analyses, we only used the HLA-G 3'UTR 14-bp allele frequency data. Some articles appeared in more than one analysis because these studies presented data for different groups of patients or diseases/ clinical situations. We firstly analyzed the two large groups (A and B). Subsequently, we evaluated the six groups described above (1 to 6 ). The main meta-analysis data obtained are presented in Table 1 . Due to diseases similar characteristics, we analyzed autoimmune and inflammatory diseases groups together, but we didn't find significative differences from the separated groups analysis $(-14 \mathrm{bp}$; OR $=1.04$; $95 \% \mathrm{CI}=0.96-1.12 ; \mathrm{p}=0.385)$. This also happened when we stratified the Transplant Rejection group in four subgroups for comparisons: solid transplants $(+14 \mathrm{pb} ; \mathrm{OR}=1.23 ; 95 \% \mathrm{CI}=0.77-1.98 ; \mathrm{p}=0.385)$, cell transplants $\quad(\mathrm{OR}=1.04 ; \quad 95 \% \mathrm{CI}=0.84-1.29 ; \mathrm{p}=0.717)$, patients against donors $(+14 \mathrm{bp} ; \mathrm{OR}=1.20 ; 95 \% \mathrm{CI}=0.69-2.11 ; \mathrm{p}=0.514)$ and transplants with or without rejection $(+14 \mathrm{bp}$; OR $=1.10$; $95 \% \mathrm{CI}=0.72-1.69 ; \mathrm{p}=0.655)$. Statistic data from these supplementary analyses are not showed in Table 1 . A meta-analysis was also performed for each disease/clinical situation with three or more published studies (Breast cancer, Cervical cancer, Hepatocellular carcinoma, Multiple sclerosis, Rheumatoid arthritis, Systemic lupus erythematosus, Type 1 diabetes, HIV vertical transmission - mothers, Human cytomegalovirus infection, Human papillomavirus infection, Heart transplantation, Kidney transplantation, Preeclampsia - when maternal alleles were evaluated, Preeclampsia - when children alleles were evaluated, Implantation failure, and Miscarriage). Considering the presence of $H L A-G$ 3'UTR 14-bp as a risk factor, we found significant results for the group called Reproductive Disorders, which includes

\footnotetext{
${ }^{4}$ García-González et al., 2014 [69] and Glas et al., 2007 [77] appear two times in this group, because two distinct diseases data are described in each article.

${ }^{5}$ Waterhouse et al., 2013 [] appears two times in this group, because two distinct groups are described in the same article.
} 


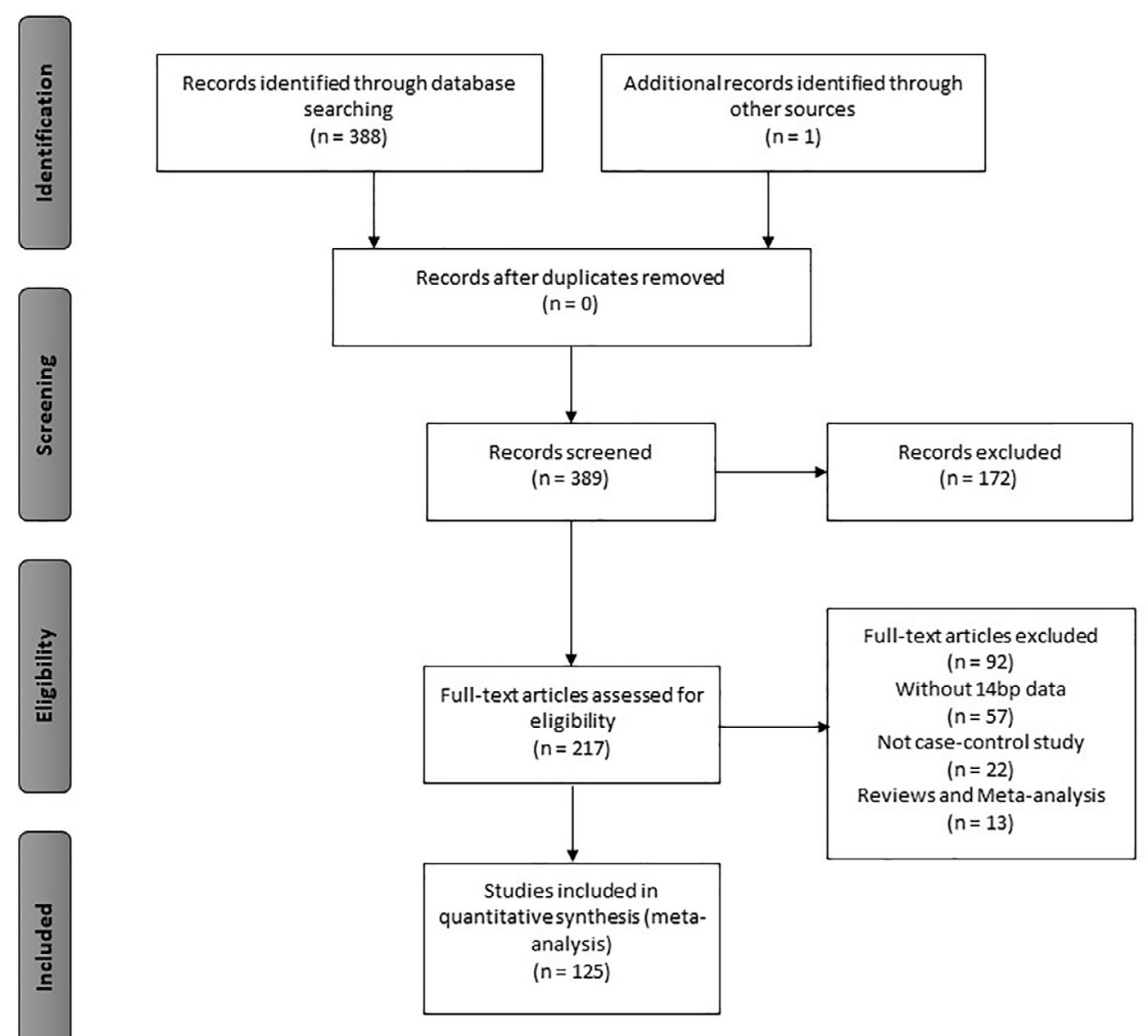

Fig. 1. Flowchart illustrating the search strategy used to identify relevant studies.

studies about Implantation Failure, Preeclampsia, Recurrent Miscarriage, Reproductive Failure and Spontaneous Miscarriage (Fig. 2); and for the following diseases analyzed individually: Preeclampsia when children alleles were evaluated (Fig. 3A) and Systemic Lupus Erythematosus (Fig. 3B). On the other hand, considering the absence of HLA-G 3'UTR 14-bp as a risk factor, we observed significant results only for two conditions analyzed separately: Breast cancer (Fig. 4A) and Human cytomegalovirus infection (Fig. 4B). Despite our main purpose was to perform a global analysis, we took into account the genetics differences between worldwide populations and we reanalyzed all data excluding specific populations groups that could interfere in the results obtained (e.g. Chinese Han population). However, we didn't observe significative differences excluding these populations (data not showed). The only exception was the Human cytomegalovirus infection metaanalysis, in which two of the three publications included were about Chinese Han population. Finally, about publication bias, we just verified a significant result in Autoimmune Diseases group meta-analysis $(\mathrm{p}=0.0009)$.

\subsection{Associations between the 14-bp polymorphism alleles and HLA-G promoters, coding regions, 3'UTRs haplotypes and proteins}

To better understand the relations between the 14-bp polymorphism and others HLA-G polymorphic sites, we organized Table 2, which contains the main associations between the 14-bp polymorphism alleles and the more frequent $H L A-G$ promoters, coding regions, $3^{\prime}$ UTRs haplotypes and proteins. In the first column are indicated the two alleles of 14-bp polymorphism (absence/deletion and presence/insertion). In the second column are classified the most frequent UTRs for each allele. The third column describes the more frequent $H L A-G$ promoter haplotypes and we could highlight that, the haplotypes belonging to the same haplotype groups appear associated with the both 14-bp alleles (e.g. promoter 0104 with absence of 14-bp and promoter 0102 and presence of 14-bp). In the fourth column are described the most frequent coding region haplotypes with each 14-bp alleles. And, in the last column are listed the most frequent proteins, where we can observe that the $\mathrm{G} * 01: 01$ protein is the most frequent for both 14-bp alleles. In addition, although different proteins are associated with the 14-bp alleles, their frequencies are generally low and studies show that these variants maintain the HLA-G functions.

\section{Discussion}

In this systematic review followed by meta-analysis, we observed a substantial number of published articles (125) that had studied, as a major objective, the association between the HLA-G 14-bp polymorphism (presence or absence) with many different disorders. We believe that among the main reasons for the large number of studies is the assumption that the 14-bp polymorphism can be directly related with HLA-G expression levels. Since the alternative splicing that happens in this region can generate complete sequences as well as sequences with 92-bp deletion, distinct HLA-G expression levels could be observed [6,10-12]. It is also important to emphasize that, the 14-bp polymorphism is easily detected by a simple and cheap technique, 
Table 1

Combined results of all meta-analyses performed in the present study.

\begin{tabular}{|c|c|c|c|c|c|c|c|c|c|c|c|c|c|}
\hline \multirow{2}{*}{$\begin{array}{l}\text { Group of diseases or } \\
\text { clinical situations }\end{array}$} & \multirow{2}{*}{$\begin{array}{l}14 \mathrm{bp} \\
\text { Allele }\end{array}$} & \multicolumn{4}{|l|}{ Number } & \multicolumn{3}{|c|}{ Fixed Model } & \multicolumn{3}{|c|}{ Random Model } & \multirow{2}{*}{$\begin{array}{l}\text { Heterogeneity } \\
-\left(\mathrm{I}^{2}, \tau_{2}, p\right. \\
\text { values })\end{array}$} & \multirow{2}{*}{$\begin{array}{l}\text { Publication Bias } \\
\text { Egger's Test }\end{array}$} \\
\hline & & Studies & $\begin{array}{l}\text { Diseases or } \\
\text { clinical } \\
\text { situations }\end{array}$ & Cases & Controls & OR & IC & $\mathrm{p}$ & OR & IC & $\mathrm{p}$ & & \\
\hline $\begin{array}{l}\text { Diseases or clinical } \\
\text { situations with } \\
\text { hypothetical high } \\
\text { HLA-G expression }\end{array}$ & $\begin{array}{l}\text { Del } \\
\text { Ins }\end{array}$ & 32 & 20 & 6091 & 8687 & & & & $\begin{array}{l}1.09 \\
0.92\end{array}$ & $\begin{array}{l}0.98-1.21 \\
0.83-1.02\end{array}$ & $\begin{array}{l}0.097 \\
0.097\end{array}$ & $\begin{array}{l}74 \%, 0.072 \\
<0.01\end{array}$ & 0.3109 \\
\hline $\begin{array}{l}\text { Diseases or clinical } \\
\text { situations with } \\
\text { hypothetical low } \\
\text { HLA-G expression }\end{array}$ & $\begin{array}{l}\text { Del } \\
\text { Ins }\end{array}$ & 107 & 32 & 16,641 & 20,721 & & & & $\begin{array}{l}0.98 \\
1.02\end{array}$ & $\begin{array}{l}0.92-1.05 \\
0.95-1.09\end{array}$ & $\begin{array}{l}0.617 \\
0.620\end{array}$ & $\begin{array}{l}75 \%, 0.091 \\
<0.01\end{array}$ & 0.2346 \\
\hline Cancer & $\begin{array}{l}\text { Del } \\
\text { Ins }\end{array}$ & 21 & 11 & 3815 & 5802 & & & & $\begin{array}{l}0.94 \\
1.06\end{array}$ & $\begin{array}{l}0.84-1.05 \\
0.95-1.19\end{array}$ & $\begin{array}{l}0.312 \\
0.312\end{array}$ & $\begin{array}{l}68 \%, 0.046 \\
<0.01\end{array}$ & 0.5915 \\
\hline Autoimmune diseases & $\begin{array}{l}\text { Del } \\
\text { Ins }\end{array}$ & 27 & 8 & 6008 & 6866 & & & & $\begin{array}{l}0.99 \\
1.01\end{array}$ & $\begin{array}{l}0.92-1.08 \\
0.93-1.09\end{array}$ & $\begin{array}{l}0.089 \\
0.089\end{array}$ & $\begin{array}{l}55 \%, 0.024 \\
<0.01\end{array}$ & 0.0009 \\
\hline Inflammatory diseases & $\begin{array}{l}\text { Del } \\
\text { Ins }\end{array}$ & 15 & 9 & 2942 & 4889 & & & & $\begin{array}{l}1.11 \\
0.90\end{array}$ & $\begin{array}{l}0.94-1.32 \\
0.76-1.07\end{array}$ & $\begin{array}{l}0.224 \\
0.225\end{array}$ & $\begin{array}{l}82 \%, 0.087 \\
<0.01\end{array}$ & 0.1447 \\
\hline Infectious diseases & $\begin{array}{l}\text { Del } \\
\text { Ins }\end{array}$ & 17 & 9 & 2276 & 2885 & & & & $\begin{array}{l}1.14 \\
0.88\end{array}$ & $\begin{array}{l}0.93-1.39 \\
0.72-1.07\end{array}$ & $\begin{array}{l}0.196 \\
0.196\end{array}$ & $\begin{array}{l}79 \%, 0.133 \\
<0.01\end{array}$ & 0.5016 \\
\hline Transplant rejection & $\begin{array}{l}\text { Del } \\
\text { Ins }\end{array}$ & 15 & 8 & 1226 & 1507 & & & & $\begin{array}{l}0.87 \\
1.15\end{array}$ & $\begin{array}{l}0.61-1.23 \\
0.81-1.64\end{array}$ & $\begin{array}{l}0.429 \\
0.429\end{array}$ & $\begin{array}{l}89 \%,<0.01 \\
0.415\end{array}$ & 0.127 \\
\hline $\begin{array}{r}\text { Reproductive } \\
\text { disorders }\end{array}$ & $\begin{array}{l}\text { Del } \\
\text { Ins }\end{array}$ & 45 & 7 & 4189 & 4574 & & & & $\begin{array}{l}0.89 \\
1.12\end{array}$ & $\begin{array}{l}0.81-0.99 \\
1.01-1.24\end{array}$ & $\begin{array}{l}0.034 \\
0.034\end{array}$ & $\begin{array}{l}58 \%, 0.065 \\
<0.01\end{array}$ & 0.701 \\
\hline Breast cancer & $\begin{array}{l}\text { Del } \\
\text { Ins }\end{array}$ & 5 & - & 726 & 811 & $\begin{array}{l}1.23 \\
0.81\end{array}$ & $\begin{array}{l}1.06-1.42 \\
0.70-0.94\end{array}$ & $\begin{array}{l}\mathbf{0 . 0 0 6} \\
0.006\end{array}$ & $\begin{array}{l}1.23 \\
0.81\end{array}$ & $\begin{array}{l}1.06-1.42 \\
0.70-0.94\end{array}$ & $\begin{array}{l}\mathbf{0 . 0 0 6} \\
0.006\end{array}$ & $0 \%, 0,0.45$ & $*$ \\
\hline Cervical cancer & $\begin{array}{l}\text { Del } \\
\text { Ins }\end{array}$ & 4 & - & 592 & 1383 & & & & $\begin{array}{l}1.04 \\
0.96\end{array}$ & $\begin{array}{l}0.78-1.38 \\
0.72-1.28\end{array}$ & $\begin{array}{l}0.787 \\
0.787\end{array}$ & $\begin{array}{l}65 \%, 0.051 \\
0.04\end{array}$ & * \\
\hline $\begin{array}{r}\text { Hepatocellular } \\
\text { carcinoma }\end{array}$ & $\begin{array}{l}\text { Del } \\
\text { Ins }\end{array}$ & 3 & - & 518 & 1071 & & & & $\begin{array}{l}1.14 \\
0.88\end{array}$ & $\begin{array}{l}0.76-1.69 \\
0.59-1.31\end{array}$ & $\begin{array}{l}0.529 \\
0.529\end{array}$ & $\begin{array}{l}79 \%, 0.096 \\
<0.01\end{array}$ & * \\
\hline Multiple sclerosis & $\begin{array}{l}\text { Del } \\
\text { Ins }\end{array}$ & 4 & - & 799 & 705 & $\begin{array}{l}0.93 \\
1.08\end{array}$ & $\begin{array}{l}0.80-1.08 \\
0.93-1.26\end{array}$ & $\begin{array}{l}0.322 \\
0.322\end{array}$ & $\begin{array}{l}0.93 \\
1.08\end{array}$ & $\begin{array}{l}0.80-1.08 \\
0.93-1.26\end{array}$ & $\begin{array}{l}0.322 \\
0.322\end{array}$ & $0 \%, 0,0.91$ & * \\
\hline Rheumatoid arthritis & $\begin{array}{l}\text { Del } \\
\text { Ins }\end{array}$ & 6 & - & 1501 & 1486 & $\begin{array}{l}1.04 \\
0.96\end{array}$ & $\begin{array}{l}0.94-1.15 \\
0.87-1.07\end{array}$ & $\begin{array}{l}0.462 \\
0.463\end{array}$ & $\begin{array}{l}1.04 \\
0.96\end{array}$ & $\begin{array}{l}0.94-1.15 \\
0.87-1.07\end{array}$ & $\begin{array}{l}0.462 \\
0.463\end{array}$ & $0 \%, 0,0.82$ & $*$ \\
\hline $\begin{array}{l}\text { Systemic lupus } \\
\text { erythematosus }\end{array}$ & $\begin{array}{l}\text { Del } \\
\text { Ins }\end{array}$ & 10 & - & 2906 & 3412 & & & & $\begin{array}{l}0.88 \\
1.13\end{array}$ & $\begin{array}{l}0.79-0.99 \\
1.01-1.27\end{array}$ & $\begin{array}{l}0.028 \\
0.028\end{array}$ & $\begin{array}{l}51 \%, 0.016 \\
0.03\end{array}$ & 0.314 \\
\hline Type 1 diabetes & $\begin{array}{l}\text { Del } \\
\text { Ins }\end{array}$ & 3 & - & 361 & 412 & $\begin{array}{l}1.24 \\
0.80\end{array}$ & $\begin{array}{l}1.01-1.52 \\
0.66-1.00\end{array}$ & $\begin{array}{l}0.036 \\
0.036\end{array}$ & $\begin{array}{l}1.24 \\
0.80\end{array}$ & $\begin{array}{l}1.01-1.52 \\
0.66-1.00\end{array}$ & $\begin{array}{l}0.049 \\
0.049\end{array}$ & $\begin{array}{l}12 \%, 0.005 \\
0.32\end{array}$ & * \\
\hline $\begin{array}{l}\text { HIV vertical } \\
\text { transmission }\end{array}$ & $\begin{array}{l}\text { Del } \\
\text { Ins }\end{array}$ & 5 & - & 468 & 702 & & & & $\begin{array}{l}1.08 \\
0.93\end{array}$ & $\begin{array}{l}0.60-1.95 \\
0.51-1.67\end{array}$ & $\begin{array}{l}0.798 \\
0.798\end{array}$ & $\begin{array}{l}88 \%, 0.386 \\
<0.01\end{array}$ & * \\
\hline $\begin{array}{l}\text { Human } \\
\text { cytomegalovirus } \\
\text { infection }\end{array}$ & $\begin{array}{l}\text { Del } \\
\text { Ins }\end{array}$ & 3 & - & 198 & 642 & $\begin{array}{l}2.06 \\
0.49\end{array}$ & $\begin{array}{l}1.60-2.64 \\
0.38-0.63\end{array}$ & $\begin{array}{l}<0.0001 \\
<0.0001\end{array}$ & $\begin{array}{l}2.06 \\
0.49\end{array}$ & $\begin{array}{l}1.60-2.64 \\
0.38-0.63\end{array}$ & $\begin{array}{l}<0.0001 \\
<0.0001\end{array}$ & $0 \%, 0,0.76$ & $*$ \\
\hline $\begin{array}{l}\text { Human papillomavirus } \\
\text { infection }\end{array}$ & $\begin{array}{l}\text { Del } \\
\text { Ins }\end{array}$ & 4 & - & 593 & 702 & & & & $\begin{array}{l}1.17 \\
0.85\end{array}$ & $\begin{array}{l}0.89-1.56 \\
0.64-1.13\end{array}$ & $\begin{array}{l}0.270 \\
0.270\end{array}$ & $\begin{array}{l}60 \%, 0.049 \\
0.06\end{array}$ & $*$ \\
\hline Heart transplantation & $\begin{array}{l}\text { Del } \\
\text { Ins }\end{array}$ & 3 & - & 337 & 261 & $\begin{array}{l}0.92 \\
1.08\end{array}$ & $\begin{array}{l}0.73-1.17 \\
0.86-1.37\end{array}$ & $\begin{array}{l}0.504 \\
0.504\end{array}$ & $\begin{array}{l}0.92 \\
1.08\end{array}$ & $\begin{array}{l}0.73-1.17 \\
0.86-1.37\end{array}$ & $\begin{array}{l}0.507 \\
0.507\end{array}$ & $0 \%, 0,0.43$ & * \\
\hline Kidney transplantation & $\begin{array}{l}\text { Del } \\
\text { Ins }\end{array}$ & 3 & - & 165 & 166 & $\begin{array}{l}1.19 \\
0.84\end{array}$ & $\begin{array}{l}0.87-1.64 \\
0.61-1.15\end{array}$ & $\begin{array}{l}0.281 \\
0.281\end{array}$ & $\begin{array}{l}1.19 \\
0.84\end{array}$ & $\begin{array}{l}0.87-1.64 \\
0.61-1.15\end{array}$ & $\begin{array}{l}0.281 \\
0.281\end{array}$ & $0 \%, 0,0.70$ & * \\
\hline Eclampsia (mothers) & $\begin{array}{l}\text { Del } \\
\text { Ins }\end{array}$ & 12 & - & 1059 & 1457 & & & & $\begin{array}{l}0.86 \\
1.16\end{array}$ & $\begin{array}{l}0.72-1.04 \\
0.96-1.39\end{array}$ & $\begin{array}{l}0.116 \\
0.116\end{array}$ & $\begin{array}{l}54 \%, 0.052 \\
<0.01\end{array}$ & 0.227 \\
\hline Eclampsia (children) & $\begin{array}{l}\text { Del } \\
\text { Ins }\end{array}$ & 3 & - & 122 & 299 & $\begin{array}{l}0.71 \\
1.42\end{array}$ & $\begin{array}{l}0.52-0.96 \\
1.04-1.93\end{array}$ & $\begin{array}{l}0.026 \\
0.026\end{array}$ & $\begin{array}{l}0.71 \\
1.42\end{array}$ & $\begin{array}{l}0.52-0.96 \\
1.04-1.93\end{array}$ & $\begin{array}{l}0.026 \\
0.026\end{array}$ & $0 \%, 0,0.70$ & $*$ \\
\hline Implantation failure & $\begin{array}{l}\text { Del } \\
\text { Ins }\end{array}$ & 4 & - & 127 & 214 & & & & $\begin{array}{l}1.38 \\
0.72\end{array}$ & $\begin{array}{l}0.60-3.18 \\
0.31-1.67\end{array}$ & $\begin{array}{l}0.447 \\
0.447\end{array}$ & $\begin{array}{l}83 \%, 0.597 \\
<0.01\end{array}$ & $*$ \\
\hline Miscarriage & $\begin{array}{l}\text { Del } \\
\text { Ins }\end{array}$ & 24 & - & 2773 & 2475 & & & & $\begin{array}{l}0.89 \\
1.12\end{array}$ & $\begin{array}{l}0.79-1.01 \\
0.99-1.27\end{array}$ & $\begin{array}{l}0.075 \\
0.077\end{array}$ & $\begin{array}{l}54 \%, 0.048 \\
<0.01\end{array}$ & 0.786 \\
\hline
\end{tabular}

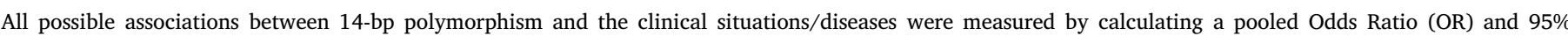

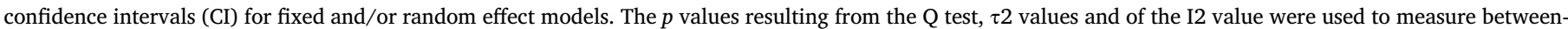

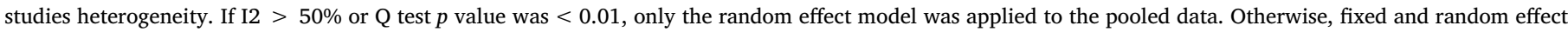

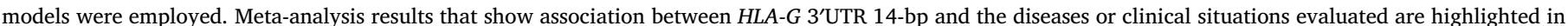
boldface. *Due to the defined parameters for this statistical test, Publication bias was not evaluated in groups with $<10$ studies.

justifying the choice of this marker for genetic studies.

The 14-bp is the most studied $H L A-G$ variation site. Notwithstanding that, this meta-analysis revealed inconsistencies between some association studies that have already been published. This statement is based on the following results: i) when we observed data from many conditions (Cervical cancer, Hepatocellular carcinoma, Multiple sclerosis, Rheumatoid arthritis, HIV vertical transmission, Human papillomavirus infection, Heart transplantation, Kidney transplantation,
Preeclampsia (mothers), Implantation failure, Miscarriage, e.g), we could verify that some studies found the 14-bp insertion associated with susceptibility to disease development [108,112,117-119,138,140], whereas, others revealed the 14-bp deletion as the allele associated with susceptibility $[47,53,79,82,91]$, and sometimes none was associated $[48,49,80,98,101,105,147]$; ii) when the disorders were grouped according to conditions exhibiting a hypothetical high (1) or low (2) HLA-G expression, no associations between the 14-bp alleles were 


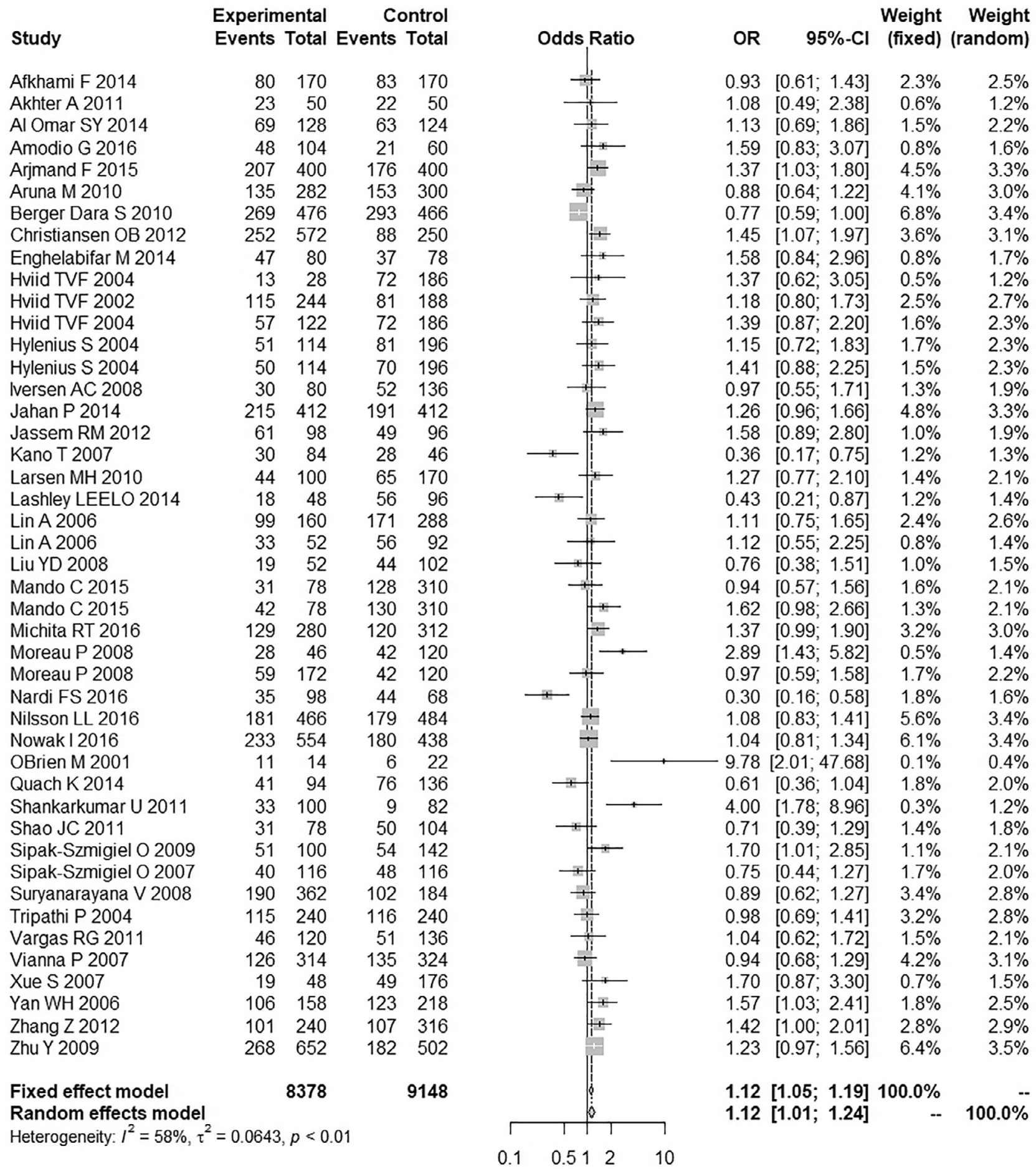

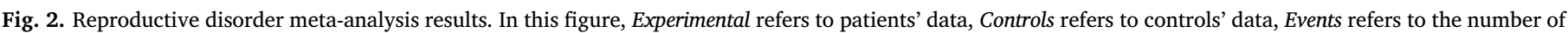

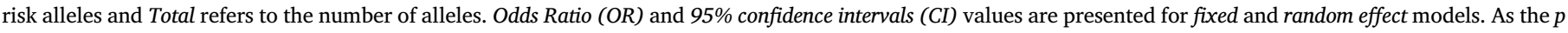
value resulting of $\mathrm{Q}$ test was $<0.01$ and $\mathrm{I} 2$ value was $>50 \%$, the random effect model was applied to these data.

observed. In other words, tumor and chronically virus-infected cells may express HLA-G to diminish or impede the action of host cytotoxic cells, and in these conditions the expression of HLA-G may be hypothetically high regardless of the individual genotype. In contrast, in autoimmune and chronic inflammatory disorders, and in engrafted organs or hematopoietic cells, the low expression of HLA-G may hypothetically be associated with poor outcome. Therefore, in these conditions, one would expect the 14-bp deletion associated with a worse outcome in the case of tumor and virus-infected cells and with a better outcome in autoimmune and in engrafted tissues. According to this meta-analysis, these associations were not observed; and iii) when we clustered diseases according to the major underlying condition; we only verified a risk association between the disease cluster called $R e$ productive Disorders (composed by studies about Implantation Failure, Preeclampsia, Recurrent Miscarriage, Reproductive Failure and Spontaneous Miscarriage) and the presence of 14-bp. Other metanalyses realized by Fan et al. (2017) and Wang et al. (2013) also verified the presence of 14-bp as a risk to implantation failure and miscarriage, 
A

Study

Hylenius, S 2004

Mando, C 2015

Fixed effect model

Random effects model

Heterogeneity: $I^{2}=0 \%, \tau^{2}=0, p=0.70$

\section{Experimental Control Events Total Events Total}

$\begin{array}{rrrr}50 & 114 & 70 & 196 \\ 33 & 52 & 56 & 92 \\ 42 & 78 & 130 & 310 \\ & & & \\ & \mathbf{2 4 4} & & \mathbf{5 9 8}\end{array}$

598

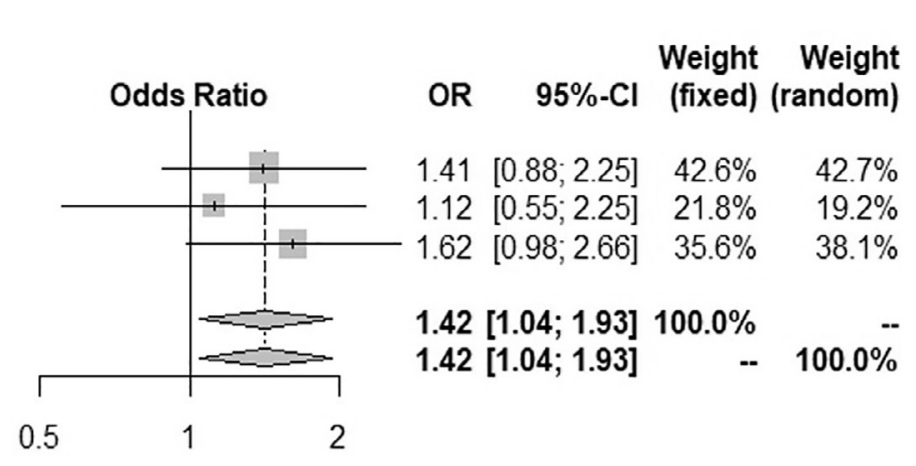

B

Study

Rizzo, R 2008

Veit, TD 2009

Wu, FX 2009

Mu, LJ 2009

Consiglio, CR 2011

Pedroza, L 2011

Lucena-Silva, N 2013

Chen, $\Pi 2013$

Catamo, E 2015

Hachiya, Y 2016

Fixed effect model

Random effects model

Heterogeneity: $I^{2}=51 \%, \tau^{2}=0.0161, p=0.03$

\section{Experimental Control Events Total Events Total}

$\begin{array}{rrr}192 & 400 & 3 \\ 242 & 586 & 3 \\ 177 & 462 & 2 \\ 342 & 972 & 1 \\ 172 & 390 & 1 \\ 55 & 166 & 2 \\ 178 & 380 & 2 \\ 196 & 542 & 1 \\ 94 & 228 & 1 \\ 450 & 1686 & 3 \\ & \\ 5812 & \\ 0.0161, p & =0.03\end{array}$

$\begin{array}{rr}351 & 902 \\ 363 & 920 \\ 289 & 734 \\ 160 & 572 \\ 102 & 244 \\ 201 & 490 \\ 223 & 566 \\ 193 & 584 \\ 109 & 256 \\ 378 & 1556 \\ & \\ & \end{array}$

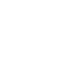

Odds Ratio

734

572 244

490

566

584 256 is

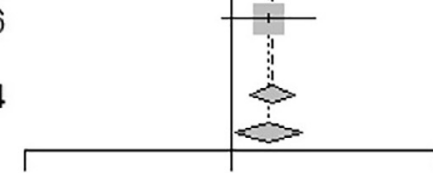

$\begin{array}{lll}0.5 & 1 & 2\end{array}$

\section{$95 \%-\mathrm{Cl}$ (fixed) (random)}

$\begin{array}{rrr}1.45[1.14 ; 1.84] & 9.0 \% & 10.7 \% \\ 1.08[0.87 ; 1.33] & 13.3 \% & 11.9 \% \\ 0.96[0.75 ; 1.21] & 11.0 \% & 10.7 \% \\ 1.40[1.12 ; 1.75] & 10.5 \% & 11.3 \% \\ 1.10[0.79 ; 1.52] & 5.6 \% & 7.6 \% \\ 0.71[0.49 ; 1.03] & 5.5 \% & 6.4 \% \\ 1.36[1.04 ; 1.76] & 7.6 \% & 9.7 \% \\ 1.15[0.90 ; 1.47] & 9.5 \% & 10.4 \% \\ 0.95[0.66 ; 1.36] & 4.8 \% & 6.6 \% \\ 1.13[0.97 ; 1.33] & 23.1 \% & 14.6 \% \\ 1.15[1.06 ; 1.24] & 100.0 \% & -- \\ 1.13[1.01 ; 1.27] & -- & 100.0 \% \\ & & \end{array}$

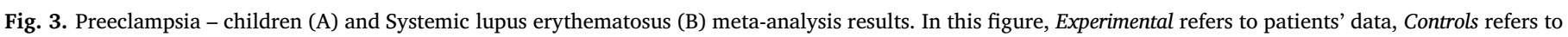

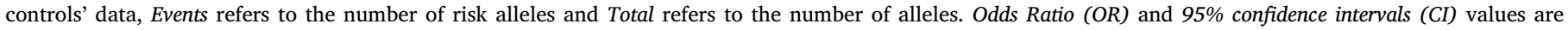

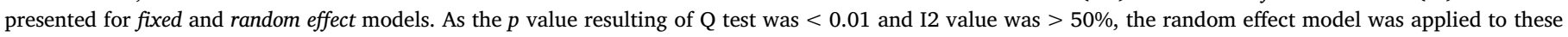
data.

respectively.

As reported at the present study, the presence of 14-bp insertion was associated with susceptibility to preeclampsia (when children alleles were evaluated) and systemic lupus erythematosus. This last data is in accordance with the metanalyses performed by Catamo et al. (2015) and Zhang et al. (2014) that described the 14-bp insertion polymorphism as a risk to Lupus too. Whereas the 14-bp deletion was associated with risk to human cytomegalovirus infection and breast cancer. The metanalysis realized by Ge et al. (2014) similarly described the 14-bp absence polymorphism as a risk factor for breast cancer in Asian populations as a result of a cancer metanalysis.

Taken together, these findings indicate that the 14-bp variability at the HLA-G 3'UTR segment is not an efficient genetic marker for disease association studies. We must consider that these are complex diseases and, although the presence of HLA-G was already described in most of these pathologies, this is probably not a direct cause-and-effect relation. Besides that, the 14-bp variation site is not the only element responsible for the posttranscriptional control of the HLA-G expression. As previously mentioned, in addition to the 14-bp insertion (associated with decreased mRNA expression) at least two other 3'UTR variation sites $(+3142 \mathrm{C} / \mathrm{G}$ and $+3187 \mathrm{~A} / \mathrm{G})[8,9]$ have been functionally studied. The $+3142 \mathrm{G}$ (associated with decreased expression due to the action of particular microRNAs) and +3187A (decreased stability of the mRNA due to an AU-rich motif) alleles have been associated with decreased HLA-G expression. Besides, other variation sites $(+3003 \mathrm{C} / \mathrm{G},+3010$ $\mathrm{G} / \mathrm{C},+3027 \mathrm{C} / \mathrm{A},+3035 \mathrm{C} / \mathrm{T},+3196 \mathrm{C} / \mathrm{G})$, which have not been functionally studied, do exhibit in silico evidences for their putative posttranscriptional HLA-G modulation [6,148,149].

Another evidence based on the association of the HLA-G $3^{\prime} \mathrm{UTR}$ variability and the levels of soluble HLA-G indicates that the 14-bp, $+3010 \mathrm{G} / \mathrm{C},+3027 \mathrm{C} / \mathrm{A}$ and $+3035 \mathrm{C} / \mathrm{T}$ polymorphic sites have been associated with HLA-G expression [5]. Taken together, at least four of the nine variation sites commonly observed in worldwide populations may exert alone or in combination their effect on post-transcriptional regulation of HLA-G expression. Therefore, instead of genotyping just the 14-bp variation site, it would be more reasonable to type the entire 3'UTR segment to study the influence of the $H L A-G 3^{\prime}$ UTR association with diseases. Besides that, to better understand $H L A-G$ expression it is also necessary to take into consideration the gene transcriptional regulation, that is determined mostly by the promoter region. Once it is no use to have an efficient post-transcriptional regulation mechanism if there is no gene transcription. Additional studies, as in vitro assays, could help to understand the role of HLA-G regulation regions polymorphisms and how they could affect gene interactions with transcriptional factors and miRNA and, consequently, the gene expression. It is also important to consider the $H L A-G$ intense linkage disequilibrium, that could eventually justify some of associations observed between the 14-bp alleles and several diseases. Many associations observed in the studies evaluated at the present meta-analysis could be a reflection of another HLA-G polymorphic site with high linkage disequilibrium.

To better understand the relations between the 14-bp polymorphism 
A

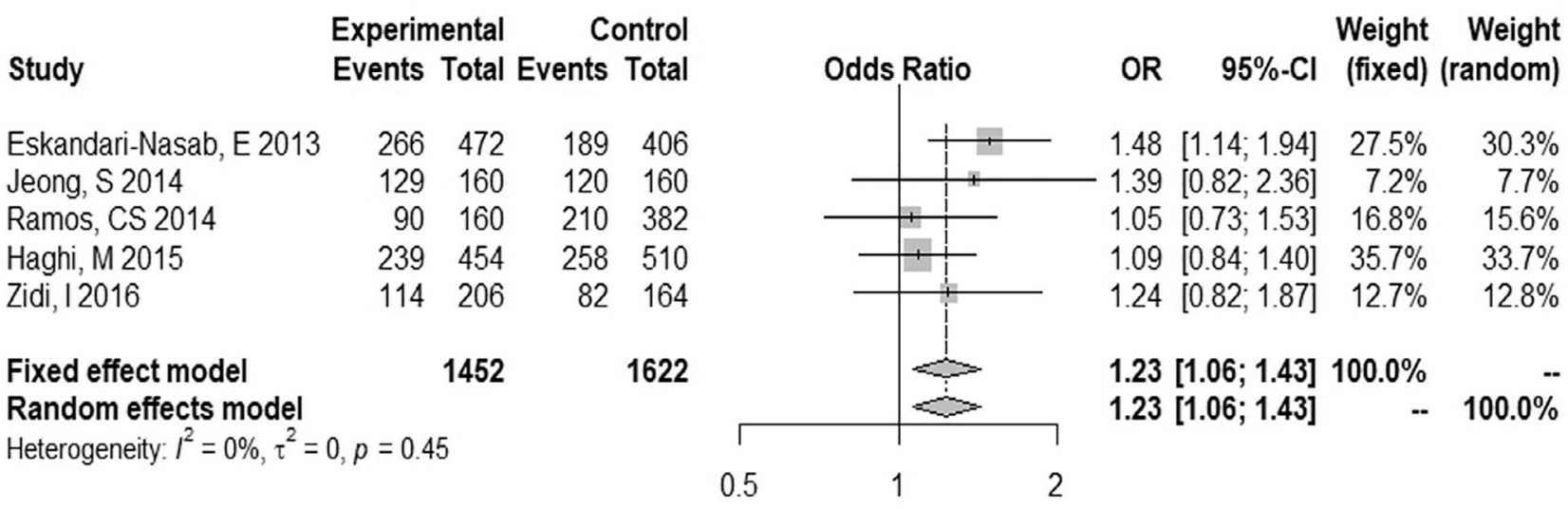

B

$\begin{array}{ll} & \text { Experimental Control } \\ \text { Study } & \text { Events Total Events Total }\end{array}$

$\begin{array}{lrrrr}\text { Zheng, ZQ 2009 } & 164 & 216 & 386 & 660 \\ \text { Jin, ZK 2012 } & 63 & 86 & 213 & 352 \\ \text { Misra, MK 2013 } & 64 & 94 & 142 & 272 \\ & & & & \\ \text { Fixed effect model } & & 396 & & \mathbf{1 2 8 4}\end{array}$

Random effects model 1284

Heterogeneity: $I^{2}=0 \%, \tau^{2}=0, p=0.76$

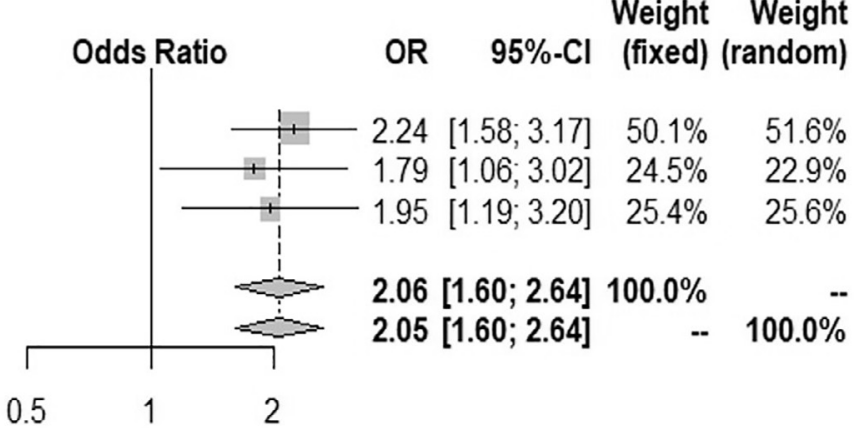

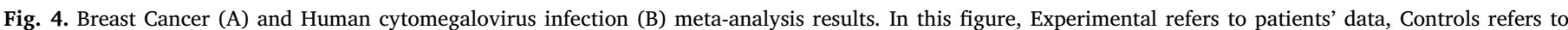

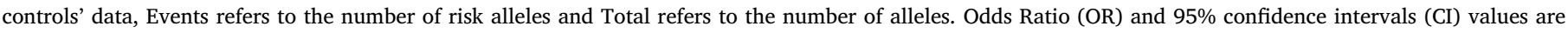

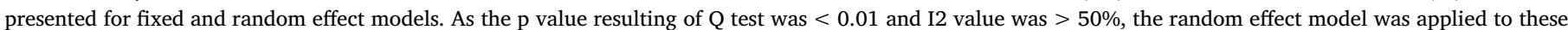
data.

Table 2

Main associations between the 14-bp polymorphism alleles and the more frequent $H L A-G$ promoters, coding regions, 3'UTRs haplotypes and proteins.

\begin{tabular}{cllll}
\hline 14-bp & $\begin{array}{l}\text { HLA-G } \\
\text { 3'UTR }\end{array}$ & $\begin{array}{l}\text { HLA-G } \\
\text { Promoter }\end{array}$ & $\begin{array}{l}\text { HLA-G } \\
\text { Coding Region }\end{array}$ & HLA-G Protein \\
\hline 14-bp & UTR-1 & 010101a & $\mathrm{G}^{*} 01: 01: 01: 01$ & $\mathrm{G}^{*} 01: 01$ \\
absence (deletion) & UTR-3 & $010101 \mathrm{~b}$ & $\mathrm{G}^{*} 01: 01: 01: 04$ & $\mathrm{G}^{*} 01: 04$ \\
& UTR-4 & $010101 \mathrm{c}$ & $\mathrm{G}^{*} 01: 01: 01: 05$ & \\
& UTR-6 & $010101 \mathrm{~d}$ & $\mathrm{G}^{*} 01: 04: 01$ & \\
& UTR-18 & $010101 \mathrm{f}$ & $\mathrm{G}^{*} 01: 04: 04$ & \\
& & $0104 \mathrm{a}$ & & \\
& & $0104 \mathrm{~b}$ & & \\
14-bp presence & UTR-2 & $010102 \mathrm{a}$ & $\mathrm{G}^{*} 01: 01: 02: 01$ & $\mathrm{G}^{*} 01: 01$ \\
(insertion) & UTR-5 & $0103 \mathrm{a}$ & $\mathrm{G}^{*} 01: 03: 01: 01$ & $\mathrm{G}^{*} 01: 03$ \\
& UTR-7 & $0103 \mathrm{~d}$ & $\mathrm{G}^{*} 01: 01: 03: 01$ & $\mathrm{G}^{*} 01: 05 \mathrm{~N}$ \\
& & & $\mathrm{G}^{*} 01: 05 \mathrm{~N}$ & $\mathrm{G}^{*} 01: 06$
\end{tabular}

and others $H L A-G$ polymorphic sites, we organized Table 2, which contains the main associations between the 14-bp polymorphism alleles and the more frequent $H L A-G$ promoters, coding regions, $3^{\prime}$ UTRs haplotypes and proteins. Considering the entire table data, we realized that the 14-bp polymorphism is not a good marker to understand the different $H L A-G$ expressions, neither to evaluate the gene function, once there are high similarities between the most frequent haplotypes for both 14-bp alleles.

Finally, although some associations were observed, the lesson learned from this meta-analysis is that the HLA-G 3'UTR 14-bp indel should be analyzed in the context of the entire 3'UTR or even the entire gene, since this whole region is under a strong linkage disequilibrium [150]. This is the first meta-analysis including a global data analysis of all disease association studies already published focusing the HLA-G 14bp polymorphism as target. As previously mentioned, the others available meta-analyses evaluated this $3^{\prime}$ UTR gene variability focusing on only one disease [14-22,151] or a small group of diseases [23-25]. Considering the meta-analysis performed in the present studied, we can conclude that, although 14-bp HLA-G $3^{\prime}$ UTR polymorphism is an easily detected variation site widely used in disease association studies, this polymorphism cannot be used as a sole genetic marker for disease susceptibility.

\section{Acknowledgements}

We acknowledge Conselho Nacional de Desenvolvimento Científico e Tecnológico (CNPq/Brazil), Coordenação de Aperfeiçoamento de Pessoal de Nível Superior (CAPES/Brazil), and Universidade de São Paulo, Faculdade de Medicina de Ribeirão Preto (FMRP/USP/Brazil) for their financial support.

\section{Conflict of interest}

The authors declare no conflicts of interest in the research. The funders had no role in study design, data collection and analysis, decision to publish or preparation of the manuscript. 


\section{Funding sources}

Conselho Nacional de Desenvolvimento Científico e Tecnológico (CNPq/Brazil), Coordenação de Aperfeiçoamento de Pessoal de Nível Superior (CAPES/Brazil), and Universidade de São Paulo, Faculdade de Medicina de Ribeirão Preto (FMRP/USP/Brazil).

\section{Appendix A. Supplementary data}

Supplementary data associated with this article can be found, in the online version, at https://doi.org/10.1016/j.humimm.2018.08.003.

\section{References}

[1] D.E. Geraghty, B.H. Koller, H.T. Orr, A human major histocompatibility complex class I gene that encodes a protein with a shortened cytoplasmic segment, PNAS 84 (1987) 9145-9149.

[2] E.A. Donadi, E.C. Castelli, A. Arnaiz-Villena, M. Roger, D. Rey, P. Moreau, Implications of the polymorphism of HLA-G on its function, regulation, evolution and disease association, Cell. Mol. Life Sci. 68 (2011) 369-395, https://doi.org/ 10.1007/s00018-010-0580-7.

[3] E.D. Carosella, N. Rouas-freiss, D.T. Roux, P. Moreau, J. Lemaout, HLA-G: An Immune Checkpoint Molecule, 1st ed., Elsevier Inc., 2015, , https://doi.org/10. 1016/bs.ai.2015.04.001.

[4] F. Morandi, R. Rizzo, E. Fainardi, N. Rouas-Freiss, V. Pistoia, Recent advances in our understanding of HLA-G biology: lessons from a wide spectrum of human diseases, J. Immunol. Res. 2016 (2016) 1-14.

[5] G. Martelli-Palomino, J.A. Pancotto, Y.C. Muniz, C.T. Mendes-Junior, E.C. Castelli, J.D. Massaro, et al., Polymorphic sites at the 3' untranslated region of the HLA-G gene are associated with differential hla-g soluble levels in the Brazilian and French population, PLoS ONE 8 (2013) 1-10, https://doi.org/10.1371/journal. pone. 0071742 .

[6] E.C. Castelli, L.C. Veiga-Castelli, L. Yaghi, P. Moreau, E.A. Donadi, Transcriptional and posttranscriptional regulations of the HLA-G gene, J. Immunol. Res. 2014 (2014) 1-14, https://doi.org/10.1155/2014/734068.

[7] A. Sabbagh, P. Luisi, E.C. Castelli, L. Gineau, D. Courtin, J. Milet, et al., Worldwide genetic variation at the 3'untranslated region of the HLA-G gene: balancing selection influencing genetic diversity, Genes Immun. 15 (2014) 95-106, https:// doi.org/10.1038/gene.2013.67.

[8] Z. Tan, G. Randall, J. Fan, B. Camoretti-Mercado, R. Brockman-Schneider, L. Pan, et al., Allele-specific targeting of microRNAs to HLA-G and risk of asthma, Am. J. Hum. Genet. 81 (2007) 829-834, https://doi.org/10.1086/521200.

[9] T.D. Veit, J.A.B. Chies, Tolerance versus immune response - MicroRNAs as important elements in the regulation of the HLA-G gene expression, Transpl. Immunol. 20 (2009) 229-231, https://doi.org/10.1016/j.trim.2008.11.001.

[10] G.A. Harrison, K.E. Humphrey, I.B. Jakobsen, D.W. Cooper, A 14 bp deletion polymorphism in the HLA-G gene, Hum. Mol. Genet. 2 (1993) 2200.

[11] T. Hviid, S. Hylenius, C. Rørbye, L. Nielsen, HLA-G allelic variants are associated with differences in the HLA-G mRNA isoform profile and HLA-G mRNA levels, Immunogenetics 55 (2003) 63-79, https://doi.org/10.1007/s00251-003-0547-z.

[12] T.V.F. Hviid, R. Rizzo, L. Melchiorri, M. Stignani, O.R. Baricordi, Polymorphism in the 5' upstream regulatory and 3' untranslated regions of the HLA-G gene in relation to soluble HLA-G and IL-10 expression, Hum. Immunol. 67 (2006) 53-62, https://doi.org/10.1016/j.humimm.2005.12.003.

[13] P. Rousseau, M. Le Discorde, G. Mouillot, C. Marcou, E.D. Carosella, P. Moreau, The 14 bp deletion-insertion polymorphism in the 3' UT Region of the HLA-G gene influences HLA-G mRNA stability, Hum. Immunol. 64 (2003) 1005-1010, https:// doi.org/10.1016/j.humimm.2003.08.347.

[14] E. Catamo, C. Addobbati, L. Segat, T. Sotero Fragoso, A. Tavares Dantas, H. de Ataide Mariz, et al., Comprehensive analysis of polymorphisms in the HLA-G 5' upstream regulatory and 3' untranslated regions in Brazilian patients with systemic lupus erythematosus, Tissue Antigens. 85 (2015) 458-465, https://doi.org/ $10.1111 / \tan .12545$.

[15] A.V.C. Coelho, R.R. Moura, S. Crovella, F. Celsi, HLA-G genetic variants and hepatocellular carcinoma: a meta-analysis, Genet. Mol. Res. 15 (2016) 1-9.

[16] W. Fan, S. Li, Z. Huang, Q. Chen, Relationship between HLA-G polymorphism and susceptibility to recurrent miscarriage: a meta-analysis of non-family-based studies, J. Assist. Reprod. Genet. 31 (2014) 173-184, https://doi.org/10.1007/ s10815-013-0155-2.

[17] W. Fan, Z. Huang, S. Li, Z. Xiao, The HLA-G 14-bp polymorphism and recurrent implantation failure : a meta-analysis, J. Assist. Reprod. Genet. 34 (2017) 1559-1565, https://doi.org/10.1007/s10815-017-0994-3.

[18] Y. Lee, S. Bae, G. Song, Meta-analysis of associations between functional HLA-G polymorphisms and susceptibility to systemic lupus erythematosus and rheumatoid arthritis, Rheumatol. Int. 35 (2015) 953-961, https://doi.org/10.1007/ s00296-014-3155-3.

[19] N. Pabalan, H. Jarjanazi, C. Sun, A.C. Iversen, Meta-analysis of the human leukocyte antigen-G (HLA-G) 14 bp insertion/deletion polymorphism as a risk factor for preeclampsia, Tissue Antigens 86 (2015) 186-194, https://doi.org/10.1111/ $\tan .12627$.

[20] X. Shi, X. Xie, Y. Jia, S. Li, Maternal genetic polymorphisms and unexplained recurrent miscarriage: a systematic review and meta-analysis, Clin. Genet. 91 (2017) 265-284.

[21] X. Wang, W. Jiang, D. Zhang, Association of 14-bp insertion/deletion polymorphism of HLA-G gene with unexplained recurrent spontaneous abortion: a meta-analysis, Tissue Antigens 81 (2013) 108-115, https://doi.org/10.1111/tan. 12056.

[22] X. Zhang, S. Li, Y. Zhang, Y. Lu, J. Wang, J. Xu, et al., Meta-analysis of the relationship between 14bp insertion/deletion polymorphism of HLA-G gene and susceptibility to systemic lupus erythematosus, Hum. Immunol. 75 (2014) 1171-1176, https://doi.org/10.1016/j.humimm.2014.10.008.

[23] Y. Ge, Q. Ge, M. Li, G. Shi, X. Xu, L. Xu, et al., Association between human leukocyte antigen - G 14-bp insertion/deletion polymorphism and cancer risk: A meta-analysis and systematic review, Hum. Immunol. 75 (2014) 827-832, https:// doi.org/10.1016/j.humimm.2014.06.004.

[24] S.K. Kim, K.H. Jeong, I.J. Kang, J.H. Chung, M.K. Shin, M.H. Lee, Relationship between the HLA-G 14 bp insertion/deletion polymorphism and susceptibility to autoimmune disease: a meta-analysis, Genet. Mol. Res. 14 (2015) 15839-15847.

[25] T. Li, H. Huang, D. Liao, H. Ling, Genetic polymorphism in HLA-G 3'UTR 14-bp ins/del and risk of cancer: a meta-analysis of case-control study, Mol. Genet. Genomics 290 (2015) 1235-1245, https://doi.org/10.1007/s00438-014-0985-3.

[26] G. Schwarzer, J.R. Carpenter, G. Rücker, Meta-Analysis with R, 2015.

[27] M. Bielska, M. Bojo, G. Klimkiewicz-wojciechowska, D. Jesionek-kupnicka, M. Borowiec, E. Kalinka-warzocha, et al., Human leukocyte antigen-G polymorphisms influence the clinical outcome in diffuse large B-cell lymphoma, Genes Chromosom. Cancer 54 (2015) 185-193, https://doi.org/10.1002/gcc.

[28] E. Eskandari-Nasab, M. Hashemi, S. Hasani, M. Omrani, M. Taheri, M. Mashhadi, Association between HLA-G 3'UTR 14-bp ins/del polymorphism and susceptibility to breast cancer, Cancer Biomark. 13 (2013) 253-259, https://doi.org/10.3233/ CBM-130364.

[29] S. Jeong, S. Park, B. Park, Y. Park, O. Kwon, H. Kim, Human leukocyte antigen-G (HLA-G) polymorphism and expression in breast cancer patients, PLoS One 9 (2014) e98284, , https://doi.org/10.1371/journal.pone.0098284.

[30] M. Haghi, M. Ali, H. Feizi, M. Sadeghizadeh, S. Lotfi, 14-bp insertion/deletion polymorphism of the HLA-G gene in breast cancer among women from North Western Iran, Asian Pac. J. Cancer Prev. 16 (2015) 6155-6158.

[31] C.S. Ramos, A.S. Gonçalves, L.C. Marinho, M.A.A. Gomes, V.A. Saddi, A.C. Lopes, et al., Analysis of HLA-G gene polymorphism and protein expression in invasive breast ductal carcinoma, Hum. Immunol. 75 (2014) 667-672, https://doi.org/10. 1016/j.humimm.2014.04.005.

[32] I. Zidi, O. Dziri, N. Zidi, R. Sebai, N. Boujelebene, W. Babay, et al., Association of HLA-G + 3142 CG polymorphism and breast cancer in Tunisian population, Immunol. Res. 64 (2016) 961-968, https://doi.org/10.1007/s12026-015-8782-6.

[33] R. Ferguson, A.V. Ramanakumar, A. Koushik, E. Franco, M. Roger, Human leukocyte antigen $\mathrm{G}$ polymorphism is associated with an increased risk of invasive cancer of the uterine cervix, Int. J. Cancer 131 (2012) 312-319, https://doi.org/ 10.1002/ijc. 27356.

[34] I.D. Silva, Y.C.N. Muniz, M.C.P.S. Sousa, K.R. Silva, E.C. Castelli, J.C.G. Filho, et al., HLA-G 3'UTR polymorphisms in high grade and invasive cervico-vaginal cancer, Hum. Immunol. 74 (2013) 452-458, https://doi.org/10.1016/j.humimm. 2012.11.025.

[35] Y. Yang, T. Chang, T. Chen, W. Lin, S. Chang, Y. Lee, Human leucocyte antigen-G polymorphisms are associated with cervical squamous cell carcinoma risk in Taiwanese women, Eur. J. Cancer 50 (2014) 469-474, https://doi.org/10.1016/j. ejca.2013.10.018.

[36] D. Bortolotti, V. Gentili, A. Rotola, D. Di Luca, R. Rizzo, Implication of HLA-G 3' untranslated region polymorphisms in human papillomavirus infection, Tissue Antigens 83 (2014) 113-118, https://doi.org/10.1111/tan.12281.

[37] M. Garziera, E. Catamo, S. Crovella, M. Montico, E. Cecchin, S. Lonardi, et al., Association of the HLA-G 3'UTR polymorphisms with colorectal cancer in Italy: a first insight, Int. J. Immunogenet. 43 (2016) 32-39, https://doi.org/10.1111/iji. 12243.

[38] Y. Chen, X. Gao, Y. Deng, H. Zhang, Relationship between HLA-G gene polymorphism and the susceptibility of esophageal cancer in Kazakh and Han nationality in Xinjiang, Biomarkers 17 (2012) 9-15, https://doi.org/10.3109/ 1354750X.2011.633242.

[39] Y. Jiang, S. Chen, S. Jia, Z. Zhu, X. Gao, Association of HLA-G 3' UTR 14-bp insertion/deletion polymorphism with hepatocellular carcinoma susceptibility in a Chinese population, DNA Cell Biol. 30 (2011) 1027-1032, https://doi.org/10. 1089/dna.2011.1238.

[40] A.C. Teixeira, F.F. Souza, L.A. Marano, N.H.S. Deghaide, S.C. Ferreira, The 14bpdeletion allele in the HLA-G gene confers susceptibility to the development of hepatocellular, Tissue Antigens 81 (2013) 408-413, https://doi.org/10.1111/tan. 12097.

[41] S.K. Kim, J.-H. Chung, J.W. Jeon, J.J. Park, J.M. Cha, K.R. Joo, et al., Association between HLA-G 14-bp insertion/deletion polymorphism and hepatocellular carcinoma in Korean patients with chronic hepatitis B viral infection, Hepatogastroenterology 60 (2013) 796-798, https://doi.org/10.5754/hge11180.

[42] V. Agnihotri, A. Gupta, R. Kumar, A. Datt, S. Dwivedi, Promising link of HLA-G polymorphism, tobacco consumption and risk of Head and Neck Squamous Cell Carcinoma (HNSCC) in North Indian population, Hum. Immunol. 78 (2017) 172-178, https://doi.org/10.1016/j.humimm.2016.12.007.

[43] A. Wisniewski, A. Kowal, E. Wyrodek, I. Nowak, E. Majorczyk, M. Wagner, Genetic polymorphisms and expression of HLA-G and its receptors, KIR2DL4 and LILRB1, in non-small cell lung cancer, Tissue Antigens 85 (2015) 466-475, https://doi. org/10.1111/tan.12561.

[44] D. T. Lau, M.D. Norris, G.M. Marshall, M. Haber, L.J. Ashton, HLA-G 
polymorphisms, genetic susceptibility, and clinical outcome in childhood neuroblastoma, Tissue Antigens 78 (2011) 421-427, https://doi.org/10.1111/j.13990039.2011.01781.x.

[45] A. Dardano, R. Rizzo, A. Polini, M. Stignani, S. Tognini, G. Pasqualetti, et al., Soluble human leukocyte antigen-g and its insertion/deletion polymorphism in papillary thyroid carcinoma: novel potential biomarkers of disease? J. Clin. Endocrinol. Metab. 97 (2016) 4080-4086, https://doi.org/10.1210/jc.2012-2231.

[46] F.M.B. Zambra, V. Biolchi, C.C.S. De Cerqueira, I.S. Brum, E.C. Castelli, J.A.B. Chies, Immunogenetics of prostate cancer and benign hyperplasia - the potential use of an HLA-G variant as a tag SNP for prostate cancer risk, HLA. 87 (2016) 79-88, https://doi.org/10.1111/tan.12741.

47] T.D. Veit, P. Vianna, I. Scheibel, C.V. Brenol, C. Brenol, J.C.T. Brenol, et al., Association of the HLA-G 14-bp insertion/deletion polymorphism with juvenile idiopathic arthritis and rheumatoid arthritis, Tissue Antigens 71 (2008) 440-446, https://doi.org/10.1111/j.1399-0039.2008.01019.x.

[48] N. Ben Fredj, K. Sakly, D. Bortolotti, M. Aissi, M. Frih-ayed, A. Rotola, et al., The association between functional HLA-G 14bp insertion/deletion and +3142 C > G polymorphisms and susceptibility to multiple sclerosis, Immunol. Lett. 180 (2016) 24-30, https://doi.org/10.1016/j.imlet.2016.10.006.

[49] A. Kroner, A. Grimm, K. Johannssen, M. Mäurer, H. Wiendl, The genetic influence of the nonclassical MHC molecule HLA-G on multiple sclerosis, Hum. Immunol. 68 (2007) 422-425, https://doi.org/10.1016/j.humimm.2007.01.012.

[50] P. Kusnierczyk, A. Wisniewski, M. Bilinska, A. Klimczak, M. Wagner, E. Majorczyk, I. Nowak, A. Pokryszko-Dragan, Association of the HLA-G gene polymorphism with multiple sclerosis in a Polish population, Int. J. Immunogenet. 37 (2010) 307-311, https://doi.org/10.1111/j.1744-313X.2010.00926.x.

[51] N. Mohammadi, M. Adib, F. Alsahebfosoul, M. Kazemi, M. Etemadifar, An investigation into the association between HLA-G 14 bp insertion/deletion poly morphism and multiple sclerosis susceptibility, J. Neuroimmunol. 290 (2016) 115-118, https://doi.org/10.1016/j.jneuroim.2015.11.019.

[52] K. Jeong, S. Kim, B. Kang, J. Chung, M. Shin, M. Lee, Association between an HLAG 14 bp insertion/deletion polymorphism and non-segmental vitiligo in the Korean population, Arch. Dermatol. Res. 306 (2014) 577-582, https://doi.org/10. 1007/s00403-014-1459-5.

[53] E. Gazit, Y. Slomov, I. Goldberg, S. Brenner, R. Loewenthal, HLA-G is associated with pemphigus vulgaris in jewish patients, Hum. Immunol. 65 (2004) 39-46, https://doi.org/10.1016/j.humimm.2003.09.019.

[54] R. Rizzo, M. Rubini, M. Govoni, M. Padovan, L. Melchiorri, M. Stignani, et al., HLA-G 14-bp polymorphism regulates the methotrexate response in rheumatoid arthritis, Pharmacogenet. Genomics 16 (2006) 615-623.

[55] T.D. Veit, C.P.S. De Lima, L.C. Cavalheiro, C.V. Brenol, J.C.T. Brenol, HLA-G +3142 polymorphism as a susceptibility marker in two rheumatoid arthritis populations in Brazil, Tissue Antigens 83 (2014) 260-266, https://doi.org/10.1111/ $\tan 12311$.

[56] E. Catamo, C. Addobbati, L. Segat, T.S. Fragoso, A.D. Barbosa, R. Jr, et al., HLA-G gene polymorphisms associated with susceptibility to rheumatoid arthritis disease and its severity in Brazilian patients, Tissue Antigens 84 (2014) 308-315, https:// doi.org/10.1111/tan.12396.

[57] C.M. Mariaselvam, A.B. Chaaben, S. Salah, D. Charron, R. Krishnamoorthy, R. Tamouza, Human leukocyte antigen-G polymorphism influences the age of onset and autoantibody status in rheumatoid arthritis, Tissue Antigens 85 (2015) 182-189, https://doi.org/10.1111/tan.12521.

[58] M. Hashemi, M. Sandoughi, S.A. Fazeli, G. Bahari, M. Rezaei, Z. Zakeri, Evaluation of HLA-G 14 bp Ins/Del and +3142G > C Polymorphism with Susceptibility and Early Disease Activity in Rheumatoid Arthritis, Adv Med. 2016 (2016) 1-7, https://doi.org/10.1155/2016/4985745.

[59] R. Rizzo, T.V.F. Hviid, M. Govoni, M. Padovan, M. Rubini, L. Melchiorri, et al., HLA-G genotype and HLA-G expression in systemic lupus erythematosus: HLA-G as a putative susceptibility gene in systemic lupus erythematosus, Tissue Antigens 71 (2008) 520-529, https://doi.org/10.1111/j.1399-0039.2008.01037.x.

[60] T. Veit, E. Cordero, T. Mucenic, O. Monticielo, J. Brenol, R. Xavier, et al., Association of the HLA-G 14 bp polymorphism with systemic lupus erythematosus, Lupus. 18 (2009) 424-430, https://doi.org/10.1177/0961203308098187.

[61] F. Wu, L. Wu, X. Luo, Z. Tang, M. Yang, C. Xie, et al., Lack of association between HLA-G 14-bp polymorphism and systemic lupus erythematosus in a Han Chinese population, Lupus. 18 (2009) 1259-1266.

[62] C.R. Consiglio, T.D. Veit, O.A. Monticielo, T. Mucenic, R.M. Xavier, J.C.T. Brenol, et al., Association of the HLA-G gene $+3142 \mathrm{C}>\mathrm{G}$ polymorphism with systemic lupus erythematosus, Tissue Antigens 77 (2011) 540-545, https://doi.org/10. 1111/j.1399-0039.2011.01635.x.

[63] L. Pedroza, M. Sauma, J.M. Vasconcelos, L.Y.C. Takeshita, E.M. Ribeiro-Rodrigues, D. Sastre, Systemic lupus erythematosus: association with KIR and SLC11A1 polymorphisms, ethnic predisposition and influence in clinical manifestations a onset revealed by ancestry genetic markers in an urban Brazilian population, Lupus. 20 (2011) 265-273.

[64] N. Lucena-silva, V. Sales, B. De Souza, R.G. Gomes, Y. Costa, N. Muniz, et al., HLAG 3' untranslated region polymorphisms are associated with systemic lupus erythematosus in 2 Brazilian populations, J. Rheumatol. 40 (2013) 1104-1113, https://doi.org/10.3899/jrheum.120814.

[65] E. Catamo, C. Addobbati, L. Segat, T.S. Fragoso, A.T. Dantas, H.D.A. Mariz, Comprehensive analysis of polymorphisms in the HLA-G 5' upstream regulatory and 3' untranslated regions in Brazilian patients with systemic lupus erythematosus, Tissue Antigens 85 (2015) 458-465, https://doi.org/10.1111/tan. 12545 .

[66] Y. Hachiya, A. Kawasaki, S. Oka, Y. Kondo, S. Ito, I. Matsumoto, et al., Association of HLA-G 3' Untranslated Region Polymorphisms with Systemic Lupus
Erythematosus in a Japanese Population: A Case-Control Association Study, PLoS ONE 11 (2016) 1-14, https://doi.org/10.1371/journal.pone.0158065.

[67] T. Chen, L. Yu, K. Huang, H. Sun, K. Lin, X. Huang, Association between HLA-G 14bp polymorphism and systemic lupus erythematosus in a Han Chinese population, Genet. Soc. China. 1 (2013).

[68] R.S. De Albuquerque, C.T. Mendes-junior, N. Lucena-silva, C. Leal, D. Meire, L.C. Veiga-castelli, et al., Association of HLA-G 30 untranslated region variants with type 1 diabetes mellitus, Hum. Immunol. 77 (2016) 358-364, https://doi. org/10.1016/j.humimm.2016.02.001.

[69] H.P.V. Silva, M.A.G. Ururahy, K.S.C. Souza, M.B. Loureiro, Y.M.C. Oliveira, G.H.M. Oliveira, et al., The association between the HLA-G 14-bp insertion / deletion polymorphism and type 1 diabetes, Genes Immun. 17 (2016) 13-18, https://doi.org/10.1038/gene.2015.45.

[70] P. Gerasimou, N. Skordis, M. Picolos, A. Spyridonidis, P. Costeas, HLA-G 14-bp polymorphism affects the age of onset in Type I Diabetes Mellitus, Int. J. Immunogenet. 43 (2016) 135-142, https://doi.org/10.1111/iji.12259.

[71] I.J. García-gonzález, Y. Valle, F. Rivas, L.E. Figuera-villanueva, J.F. Muñoz-valle, H.E. Flores-salinas, et al., The 14 bp Del/Ins HLA-G polymorphism is related with high blood pressure in acute coronary syndrome and type 2 diabetes mellitus, Biomed Res. Int. 2014 (2014) 1-8.

[72] X. Zheng, C. Li, D. Xu, A. Lin, W. Bao, G. Yang, Analysis of the plasma soluble human leukocyte antigen - $\mathrm{G}$ and interleukin-10 levels in childhood atopic asthma, HIM. 71 (2010) 982-987, https://doi.org/10.1016/j.humimm.2010.06. 018.

[73] K. Sakly, M. Maatouk, S. Hammami, O. Harzallah, W. Sakly, S. Feki, et al., HLA-G $14 \mathrm{bp}$ insertion / deletion polymorphism and its association with sHLA-G levels in Behçet's disease Tunisian patients, Hum. Immunol. 77 (2016) 90-95, https://doi. org/10.1016/j.humimm.2015.10.016.

[74] K.S. Park, J.S. Park, J.H. Nam, D. Bang, S. Sohn, E.S. Lee, HLA-E*0101 and HLA$G * 010101$ reduce the risk of Behcet's disease, Tissue Antigens 69 (2007) 139-144, https://doi.org/10.1111/j.1399-0039.2006.00742.x.

[75] E. Catamo, L. Zupin, L. Segat, F. Celsi, S. Crovella, HLA-G and susceptibility to develop celiac disease, Hum. Immunol. 76 (2015) 36-41, https://doi.org/10. 1016/j.humimm.2014.12.006.

[76] A. Fabris, L. Segat, E. Catamo, M. Morgutti, A. Vendramin, S. Crovella, HLA-G 14 bp deletion/insertion polymorphism in celiac disease, Am. J. Gastroenterol. 106 (2011) 139-144, https://doi.org/10.1038/ajg.2010.340.

[77] I. Zidi, N. Kharrat, R. Abdelhedi, A. Ben, A. Baligh, H. Ben, et al., Nonclassical human leukocyte antigen (HLA-G, HLA-E, and HLA-F) in coronary artery disease, Hum. Immunol. 77 (2016) 325-329, https://doi.org/10.1016/j.humimm.2016. 01.008.

[78] A. Lin, W. Yan, H. Xu, L. Tang, X. Chen, M. Zhu, et al., 14 bp deletion polymorphism in the HLA-G gene is a risk factor for idiopathic dilated cardiomyopathy in a Chinese Han population, Tissue Antigens 70 (2007) 427-431, https://doi.org/ 10.1111/j.1399-0039.2007.00926.x

[79] J. Glas, H. Török, L. Tonenchi, M. Wetzke, V. Beynon, M. Teshome, et al., The 14 bp deletion polymorphism in the HLA-G gene displays significant differences between ulcerative colitis and Crohn's disease and is associated with ileocecal resection in Crohn's disease, Int. Immunol. 19 (2007) 621-626, https://doi.org/10. 1093/intimm/dxm027.

[80] I. Zidi, H. Ben Yahia, D. Bortolotti, L. Mouelhi, A.B. Laaribi, S. Ayadi, et al., Int. Immunol. 27 (2015) 289-296, https://doi.org/10.1093/intimm/dxv002.

[81] Y. Liu, M. Lai, Y. Lou, Q. Han, Q. Yang, M. Chen, et al., Elevation of plasma-soluble HLA-G in childhood nephrotic syndrome is associated with IgE, Ann. Clin. Biochem. 54 (2017) 69-75, https://doi.org/10.1177/0004563216637625.

[82] M.K. Misra, S. Prakash, R. Kapoor, S.K. Pandey, R.K. Sharma, S. Agrawal, Association of HLA-G promoter and 14-bp insertion-deletion variants with acute allograft rejection and end-stage renal disease, Tissue Antigens 82 (2013) 317-326, https://doi.org/10.1111/tan.12210.

[83] C. Ferreira, S. Gama, F. Chachá, F. Fernandes, A. Corrêa, R. De Carvalho, et al., The HLA-G 14-base pair deletion allele and the deletion / deletion genotype are associated with persistent $\mathrm{HBe}$ antigenemia in chronic hepatis B infection, Hum. Immunol. 78 (2017) 166-171, https://doi.org/10.1016/j.humimm.2016.12.011.

[84] J. Genre, F.P.S. Reginaldo, J.M.D.L. Andrade, F.P. Lima, A.V. Coutinho, E.A. Donadi, et al., HLA-G 14-bp Ins/Ins Genotype in Patients Harbouring Helicobacter pylori Infection: A Potential Risk Factor? Scand. J. Immunol. 83 (2016) 52-57, https://doi.org/10.1111/sji.12390.

[85] L. Segat, E. Catamo, A. Fabris, M. Morgutti, P. D'Agaro, C. Campello, et al., HLA$\mathrm{G}^{*} 0105 \mathrm{~N}$ allele is associated with augmented risk for HIV infection in white female patients, AIDS. 31 (2010) 1961-1964, https://doi.org/10.1097/QAD. 0b013e32833c3324.

[86] H. Hong, M. Paximadis, G. Gray, L. Kuhn, C. Tiemessen, Maternal human leukocyte antigen-G (HLA-G) genetic variants associate with in utero mother-to-child transmission of HIV-1 in Black South Africans, Infect Genet Evol. 30 (2015) 147-158, https://doi.org/10.1016/j.meegid.2014.12.021.Maternal.

[87] L. Segat, L. Zupin, H. Kim, E. Catamo, D. Thea, C. Kankasa, et al., HLA-G 14 bp deletion/insertion polymorphism and mother-to-child transmission of HIV, Tissue Antigens 83 (2014) 161-167, https://doi.org/10.1111/tan.12296.HLA-G.

[88] A. Fabris, E. Catamo, L. Segat, M. Morgutti, L. Claudio, S. Crovella, Association between HLA-G 3'UTR 14-bp polymorphism and HIV vertical transmission in Brazilian children, AIDS. 23 (2009) 177-182, https://doi.org/10.1097/QAD. 0b013e32832027bf.

[89] L. Segat, E. Catamo, A. Fabris, L. Padovan, M. Morgutti, S. Crovella, HLA-G 3' UTR haplotypes and HIV vertical transmission, AIDS. 23 (2009) 1916-1918, https:// doi.org/10.1097/QAD.0b013e32832f8104.

[90] R. Haddad, D. Cilião Alves, M. Rocha-Junior, R. Azevedo, M. Pombo-de-Oliveira, 
O. Takayanagui, et al., HLA-G 14-bp insertion/deletion polymorphism is a risk factor for HTLV-1 infection, AIDS Res. Hum. Retroviruses. 27 (2011) 283-288, https://doi.org/10.1089/aid.2010.0165.

[91] X. Zheng, F. Zhu, W. Shi, A. Lin, W. Yan, The HLA-G 14 bp insertion/deletion polymorphism is a putative susceptible factor for active human cytomegalovirus infection in children, Tissue Antigens 74 (2009) 317-321, https://doi.org/10. 1111/j.1399-0039.2009.01312.x.

[92] Z. Jin, C. Xu, P. Tian, W. Xue, X. Ding, J. Zheng, Impact of HLA-G 14-bp polymorphism on acute rejection and cytomegalovirus infection in kidney transplant recipients from northwestern China, Transpl. Immunol. 27 (2012) 69-74, https:// doi.org/10.1016/j.trim.2012.06.008.

[93] H. Xu, W. Shi, A. Lin, W. Yan, HLA-G 3 ' untranslated region polymorphisms influence the susceptibility for human papillomavirus infection, Tissue Antigens 84 (2014) 216-222, https://doi.org/10.1111/tan.12359.

[94] R. Simões, M. Gonçalves, E. Castelli, C. Júnior, J. Bettini, M. Discorde, et al., HLA$\mathrm{G}$ polymorphisms in women with squamous intraepithelial lesions harboring human papillomavirus, Mod. Pathol. 22 (2009) 1075-1082, https://doi.org/10. 1038/modpathol.2009.67.

[95] N. Lucena-Silva, M.A.G. Teixeira, A.D.L. Ramos, R.S. De Albuquerque, G.T.N. Diniz, C.T. Mendes-Junior, et al., The +3187 A/G HLA-G polymorphic site is associated with polar forms and reactive reaction in leprosy, Mol. Genet. Genomic Med. 1 (2013) 123-130, https://doi.org/10.1002/mgg3.14.

[96] P. Graebin, T.D. Veit, C.S. Alho, F.S. Dias, J.A.B. Chies, Polymorphic variants in exon 8 at the 3 ' UTR of the HLA-G gene are associated with septic shock in critically ill patients, Crit. Care 16 (2012) R211, https://doi.org/10.1186/cc11845.

[97] M. Waterhouse, R. Wäsch, H. Bertz, J. Finke, Soluble HLA-G molecules and HLA-G 14-base pair polymorphism after allogeneic hematopoietic cell transplantation, TPS. 45 (2013) 397-401, https://doi.org/10.1016/j.transproceed.2012.05.073.

[98] P. Chiusolo, S. Bellesi, N. Piccirillo, S. Giammarco, S. Marietti, D. De Ritis, et al., The role of HLA - G 14-bp polymorphism in allo-HSCT after short-term course MTX for GvHD prophylaxis, Bone Marrow Transplant. 47 (2011) 120-124, https://doi.org/10.1038/bmt.2011.40.

[99] W. Boukouaci, M. Busson, C. Fortier, K. Amokrane, R. de Latour, M. Robin, et al., Association of HLA-G low expressor genotype with severe acute graft-versus-host disease after sibling bone marrow transplantation, Front. Immunol. 2 (2011) 1-6, https://doi.org/10.3389/fimmu.2011.00074.

[100] M. Torres, J. Luque, P. Lorite, B. Isla-Tejera, T. Palomeque, M. Aumente, et al., 14Base pair polymorphism of human leukocyte antigen-G as genetic determinant in heart transplantation and cyclosporine therapy monitoring, Hum. Immunol. 70 (2009) 830-835, https://doi.org/10.1016/j.humimm.2009.07.012.

[101] J. Lazarte, L. Goldraich, C. Manlhiot, S. Kozuszko, V. Rao, D. Delgado, Human leukocyte antigen-G polymorphisms association with cancer post-heart transplantation, Hum. Immunol. 77 (2016) 805-811, https://doi.org/10.1016/j. humimm.2016.01.005.

[102] H. Thude, M. Janssen, M. Sterneck, B. Nashan, M. Koch, 14-bp ins/del polymorphism and $+3142 \mathrm{C}>$ G SNP of the HLA-G gene have a significant impact on acute rejection after liver transplantation, Hum. Immunol. 77 (2016) 1159-1165, https://doi.org/10.1016/j.humimm.2016.09.009.

[103] N. Azarpira, M.H. Aghdaie, K. Kazemi, B. Geramizadeh, M. Darai, HLA-G polymorphism (rs16375) and acute rejection in liver transplant recipients, Dis. Markers 2014 (2014) 1-5, https://doi.org/10.1155/2014/814182.

[104] D.C. Cilião Alves, J.C. de Oliveira Crispim, E.C. Castelli, C.T. Mendes-Junior, N.H.S. Deghaide, G.E. Barros Silva, et al., Human leukocyte antigen-G 3' untranslated region polymorphisms are associated with better kidney allograft acceptance, Hum. Immunol. 73 (2012) 52-59, https://doi.org/10.1016/j.humimm. 2011.10.007.

[105] J. Crispim, C. Mendes-Junior, I. Wastowski, R. Costa, E. Castelli, L. Saber, et al., Frequency of insertion/deletion polymorphism in exon 8 of HLA-G and kidney allograft outcome, Tissue Antigens 71 (2008) 35-41, https://doi.org/10.1111/j. 1399-0039.2007.00961.x.

[106] M. Aghdaie, N. Azarpira, K. Kazemi, B. Geramizadeh, M. Darai, S. Malekhoseini, Frequency of HLA-G exon 8 polymorphisms and kidney allograft outcome in Iranian population, Mol. Biol. Rep. 38 (2011) 3593-3597, https://doi.org/10 1007/s11033-010-0470-y.

[107] T. Hviid, S. Hylenius, A. Lindhard, O.B. Christiansen, Association between human leukocyte antigen-G genotype and success of in vitro fertilization and pregnancy outcome, Tissue Antigens 64 (2004) 66-69, https://doi.org/10.1111/j.1399-0039. 2004.00239.x.

[108] L.E.E.L.O. Lashley, L.A.J. Van Der Westerlaken, G.W. Haasnoot, J.J.M. Drabbels, Maternal HLA-C2 and 14 bp insertion in HLA-G is associated with recurrent implantation failure after in vitro fertilization treatment, Tissue Antigens 84 (2014) 536-544, https://doi.org/10.1111/tan.12452.

[109] M. Enghelabifar, S. Allafan, J. Khayatzadeh, K.S. Abadi, M.H. Nazarabadi, F. Moradi, et al., Association of the maternal 14-bp insertion/deletion polymorphism in the histocompatibility leukocyte antigen $\mathrm{G}$ gene with recurrent implantation failure, Iran J. Reprod. Med. 12 (2014) 641-646.

[110] S. Nardi, R. Slowik, T. Michelon, L. Felipe, B. Wagner, J. Neumann, et al., High amounts of total and extracellular vesicle-derived soluble HLA-G are associated with HLA-G 14-bp deletion variant in women with embryo implantation failure, Am. J. Reprod. Immunol. 75 (2016) 661-671, https://doi.org/10.1111/aji.12507.

[111] O. Sipak-Szmigiel, C. Cybulski, D. Wokołorczyk, J. Lubiński, R. Kurzawa, T. Baczkowski, et al., HLA-G polymorphism and in vitro fertilization failure in a Polish population, Tissue Antigens 73 (2009) 348-352, https://doi.org/10.1111/j. 1399-0039.2008.01205.x.

[112] M.O. Brien, T. Mccarthy, D. Jenkins, P. Paul, J. Dausset, E.D. Carosella, et al., Altered HLA-G transcription in pre-eclampsia is associated with allele specific inheritance: possible role of the HLA-G gene in susceptibility to the disease, Cell. Mol. Life Sci. 58 (2001) 1943-1949.

[113] S. Hylenius, A.N. Andersen, M. Melbye, T.V.F. Hviid, Association between HLA-G genotype and risk of pre-eclampsia: a case-control study using family triads, Mol. Hum. Reprod. 10 (2004) 237-246, https://doi.org/10.1093/molehr/gah035.

[114] A. Lin, W.H. Yan, M.Z. Dai, X.J. Chen, B.L. Li, B.G. Chen, et al., Maternal human leukocyte antigen-G polymorphism is not associated with pre-eclampsia in a Chinese Han population, Tissue Antigens 68 (2006) 311-316, https://doi.org/10. 1111/j.1399-0039.2006.00667.x.

[115] P. Vianna, C. Dalmáz, T. Veit, C. Tedoldi, I. Roisenberg, J. Chies, Immunogenetics of pregnancy: role of a 14-bp deletion in the maternal HLA-G gene in primiparous pre-eclamptic Brazilian women, Hum. Immunol. 68 (2007) 668-674, https://doi. org/10.1016/j.humimm.2007.05.006.

[116] A. Iversen, O. Toai, D. Nguyen, L. Føll, I. Poliakova, V. Malm, et al., The HLA-G $14 \mathrm{bp}$ gene polymorphism and decidual HLA-G 14bp gene expression in preeclamptic and normal pregnancies, J. Reprod. Immunol. 78 (2008) 158-165, https://doi.org/10.1016/j.jri.2008.03.001.

[117] P. Moreau, L. Contu, F. Alba, S. Lai, R. Simoes, S. Orrù, et al., HLA-G gene polymorphism in human placentas: possible association of $\mathrm{G}^{*} 0106$ allele with preeclampsia and miscarriage, Biol. Reprod. 79 (2008) 459-467, https://doi.org/10. 1095/biolreprod.108.068874.

[118] M. Larsen, S. Hylenius, A. Andersen, T. Hviid, The 3'-untranslated region of the HLA-G gene in relation to pre-eclampsia: revisited, Tissue Antigens 75 (2010) 253-261, https://doi.org/10.1111/j.1399-0039.2009.01435.x.

[119] Z. Zhang, Y. Li, L.L. Zhang, L.T. Jia, X.Q. Yang, Association of 14 bp insertion/ deletion polymorphism of the HLA-G gene in father with severe preeclampsia in Chinese, Tissue Antigens 80 (2012) 158-164, https://doi.org/10.1111/j.13990039.2012.01907.x.

[120] P. Jahan, G. Deepthi, P. Latha, V.U. Rani, Pregnancy hypertension : an international journal of women's cardiovascular health a study on the role of HLA-G 14 bp and ACE IN / DEL polymorphisms in pre-eclamptic South Indian women, pregnancy hypertens, An Int. J. Women's Cardiovasc. Heal. 4 (2014) 164-169, https:// doi.org/10.1016/j.preghy.2014.03.002.

[121] K. Quach, S.A. Grover, S. Kenigsberg, C.L. Librach, A combination of single nucleotide polymorphisms in the 30 untranslated region of HLA-G is associated with preeclampsia, Hum. Immunol. 75 (2014) 1163-1170, https://doi.org/10.1016/j. humimm.2014.10.009.

[122] L.L. Nilsson, S. Djurisic, A.N. Andersen, M. Melbye, D. Bjerre, Distribution of HLA G extended haplotypes and one HLA-E polymorphism in a large-scale study of mother-child dyads with and without severe preeclampsia and eclampsia, HLA 88 (2016) 172-186, https://doi.org/10.1111/tan.12871.

[123] C. Mando, P. Pileri, M.I. Mazzocco, D. Lattuada, A. Zolin, M. Plebani, et al., Maternal and fetal HLA-G 14 bp gene polymorphism in pregnancy-induced hypertension, preeclampsia, intrauterine growth restricted and normal pregnancies, J. Matern Fetal Neonatal Med. 29 (2016) 1509-1514, https://doi.org/10.3109/ 14767058.2015.1052398.

[124] T. Hviid, S. Hylenius, A.M. Hoegh, C. Kruse, O.B. Christiansen, HLA-G polymorphisms in couples with recurrent spontaneous abortions, Tissue Antigens 60 (2002) 122-132.

[125] P. Tripathi, A. Abbas, S. Naik, S. Agrawal, Role of 14-bp deletion in the HLA-G gene in the maintenance of pregnancy, Tissue Antigens 64 (2004) 706-710, https://doi.org/10.1111/j.1399-0039.2004.00308.x.

[126] W.H. Yan, A. Lin, X.J. Chen, M.Z. Dai, L.H. Gan, M.Y. Zhou, et al., Association of the maternal 14-bp insertion polymorphism in the HLA-G gene in women with recurrent spontaneous abortions, Tissue Antigens 68 (2006) 521-523, https://doi org/10.1111/j.1399-0039.2006.00723.x.

[127] S. Xue, J. Yang, F. Yao, L. Xu, L. Fan, L. Fan, Recurrent spontaneous abortions patients have more $-14 \mathrm{bp} /+14 \mathrm{bp}$ heterozygotes in the 3'UT region of the HLA-G gene in a Chinese Han population, Tissue Antigens 69 (2007) 153-155, https:// doi.org/10.1111/j.1399-0039.2006.763.

[128] V. Suryanarayana, L. Rao, M. Kanakavalli, V. Padmalatha, T. Raseswari, M. Deenadayal, et al., Association between novel HLA-G genotypes and risk of recurrent miscarriages: a case-control study in a South Indian population, Reprod. Sci. 15 (2008) 817-824.

[129] Y. Zhu, Z. Huo, J. Lai, S. Li, H. Jiao, J. Dang, et al., Case-control study of a HLA-G 14-bp insertion-deletion polymorphism in women with recurrent miscarriages, Scand. J. Immunol. 71 (2010) 52-54, https://doi.org/10.1111/j.1365-3083.2009. 02348.x.

[130] M. Aruna, P.V.S. Sirisha, S.A. Bhaskar, S. Tarakeswari, K. Thangaraj, B.M. Reddy, Role of 14-bp insertion/deletion polymorphism in HLA-G among Indian women with recurrent spontaneous abortions, Tissue Antigens 77 (2011) 131-135, https://doi.org/10.1111/j.1399-0039.2010.01584.x.

[131] D.S. Berger, W.A. Hogge, M.M. Barmada, R.E. Ferrell, Comprehensive analysis of HLA-G: implications for recurrent spontaneous abortion, Reprod Sci. 17 (2010) 331-338.

[132] R. Vargas, P. Sarturi, S. Mattar, E. Bompeixe, J. Silva, A. Pirri, et al., Association of HLA-G alleles and 3' UTR 14 bp haplotypes with recurrent miscarriage in Brazilian couples, Hum. Immunol. 72 (2011) 479-485, https://doi.org/10.1016/j.humimm. 2011.02.011.

[133] A. Akhter, R.M. Faridi, V. Das, A. Pandey, S. Naik, S. Agrawal, In vitro up-regulation of HLA-G using dexamethasone and hydrocortisone in first-trimester trophoblast cells of women experiencing recurrent miscarriage, Tissue Antigens 80 (2012) 126-135, https://doi.org/10.1111/j.1399-0039.2012.01884.x.

[134] U. Shankarkumar, A. Shankarkumar, Z. Chedda, K. Ghosh, Role of 14-bp deletion/ insertion polymorphism in exon 8 of the HLA-G gene in recurrent spontaneous abortion patients, J. Hum. Reprod. Sci. 4 (2011) 143-146, https://doi.org/10. 
4103/0974-1208.92289.

[135] O.B. Christiansen, A.M. Kolte, M. Dahl, E.C. Larsen, R. Steffensen, H.S. Nielsen, et al., Maternal homozygocity for a 14 base pair insertion in exon 8 of the HLA-G gene and carriage of HLA class II alleles restricting HY immunity predispose to unexplained secondary recurrent miscarriage and low birth weight in children born to these patients, Hum. Immunol. 73 (2012) 699-705, https://doi.org/10. 1016/j.humimm.2012.04.014.

[136] R.M. Jassem, W.S. Shani, D.A. Loisel, M. Sharief, HLA-G polymorphisms and soluble HLA-G protein levels in women with recurrent pregnancy loss from Basrah province in Iraq, Hum. Immunol. 73 (2012) 811-817, https://doi.org/10.1016/j. humimm.2012.05.009.HLA-G.

[137] F. Afkhami, M.S. Khaniani, L. Farzadi, Z. Paknejad, S.M. Derakhshan, The HLA-G 14-bp insertion/deletion polymorphism in recurrent spontaneous abortion among Iranian women, Iran J. Allergy Asthma Immunol. 13 (2014) 364-369.

[138] G. Amodio, V. Canti, L. Maggio, S. Rosa, M. Teresa, P. Rovere-querini, et al., Association of genetic variants in the 30 UTR of HLA-G with recurrent pregnancy loss, Hum. Immunol. 77 (2016) 886-891, https://doi.org/10.1016/j.humimm. 2016.06.020.

[139] R.T. Michita, F.M.B. Zambra, L.R. Fraga, M.T.V. Sanseverino, S.M. CallegariJacques, P. Vianna, et al., A tug-of-war between tolerance and rejection - New evidence for 30 UTR HLA-G haplotypes influence in recurrent pregnancy loss, Hum. Immunol. 77 (2016) 892-897, https://doi.org/10.1016/j.humimm.2016. 07.004.

[140] F. Arjmand, M. Samadi, Association of 14-bp insertion/deletion polymorphism of HLA-G gene with idiopathic recurrent miscarriages in infertility center patients in Yazd, Iran, J. Immunotoxicol. 00 (2015) 1-6, https://doi.org/10.3109/1547691X. 2015.1052159.

[141] T. Kano, T. Mori, M. Furudono, H. Ishikawa, H. Watanabe, E. Kikk, Human leukocyte antigen may predict outcome of primary recurrent spontaneous abortion treated with paternal lymphocyte alloimmunization therapy, Am. J. Reprod. Immunol. 58 (2007) 383-387, https://doi.org/10.1111/j.1600-0897.2007. 00517.x.

[142] Y. Liu, J. Shao, Z. Wang, Y. Jing, D. Hu, Relationship of polymorphism of HLA-G
14 bp and RSA in the Kunmin, China, Heal. Front. 3 (2008) 4-5.

[143] J. Shao, D. Hu, J. Chen, Y. Liu, Z. Wang, Relationship of polymorphism of HLA-G $14 \mathrm{bp}$ and the expression of HLA-G mRNA with unexplained recurrent spontaneous abortion, J Pr. Obs. Gynecol. 27 (2011) 698-701.

[144] O. Sipak-Szmigiel, C. Cybulski, J. Lubin, O. Sipak-szmigiel, HLA-G polymorphism in a Polish population and reproductive failure, Tissue Antigens 71 (2007) 67-71, https://doi.org/10.1111/j.1399-0039.2007.00942.x.

[145] S.Y. Al Omar, L. Mansour, A.F. Alkhuriji, S. Alwasel, Genetic association between the HLA-G 14-bp insertion/deletion polymorphism and the recurrent spontaneous abortions in Saudi Arabian women, Genet. Mol. Res. 14 (2015) 286-293.

[146] I. Nowak, A. Malinowski, E. Barcz, J. Wilczyński, M. Wagner, E. Majorczyk, et al., Possible role of HLA-G, LILRB1 and KIR2DL4 gene polymorphisms in spontaneous miscarriage, Arch. Immunol. Ther. Exp. (Warsz) 64 (2016) 505-514, https://doi. org/10.1007/s00005-016-0389-7.

[147] T. Twito, J. Joseph, A. Mociornita, V. Rao, H. Ross, D. Delgado, The 14-bp deletion in the HLA-G gene indicates a low risk for acute cellular rejection in heart transplant recipients, J. Heart Lung Transplant. 30 (2011) 778-782, https://doi.org/10. 1016/j.healun.2011.01.726.

[148] I.O.P. Porto, C.T. Mendes-Junior, L.P. Felício, R.C. Georg, P. Moreau, E.A. Donadi, et al., microRNAs targeting the immunomodulatory HLA-G gene: A new survey searching for microRNAs with potential to regulate HLA-G, Mol. Immunol. 65 (2015) 230-241, https://doi.org/10.1016/j.molimm.2015.01.030.

[149] E.C. Castelli, P. Moreau, A.O.E. Chiromatzo, C.T. Mendes-Junior, L.C. VeigaCastelli, L. Yaghi, et al., In silico analysis of microRNAS targeting the HLA-G 3' untranslated region alleles and haplotypes, Hum. Immunol. 70 (2009) 1020-1025, https://doi.org/10.1016/j.humimm.2009.07.028.

[150] E.C. Castelli, C.T. Mendes-Junior, L.C. Veiga-Castelli, M. Roger, P. Moreau, E.A. Donadi, A comprehensive study of polymorphic sites along the HLA-G gene: implication for gene regulation and evolution, Mol. Biol. Evol. 28 (2011) 3069-3086, https://doi.org/10.1093/molbev/msr138.

[151] Z. Niu, L. Wang, R. Tk, Y. Guo, W. Sb, Y. Yao, A meta-analysis of the impact of human leukocyte antigen-G on the outcomes of IVF/ICSI, Reprod. Biomed. Online. 34 (2017) 611-618, https://doi.org/10.1016/j.rbmo.2017.03.002. 